\title{
Research on Roll Stabilizing Based on Energy Optimization for Autonomous Surface Vehicle
}

\author{
Hongjian Wang, ${ }^{1}$ Linlin Wang, ${ }^{1,2}$ and Lixin $\operatorname{Pan}^{3}$ \\ ${ }^{1}$ College of Automation, Harbin Engineering University, Harbin 150001, China \\ ${ }^{2}$ College of Information, Inner Mongolia University of Technology, Hohhot 010051, China \\ ${ }^{3}$ Beijing Institute of Control Engineering, China Academy of Space Technology, Beijing 100190, China \\ Correspondence should be addressed to Linlin Wang; willmaomao@sina.com
}

Received 11 May 2014; Revised 9 August 2014; Accepted 11 August 2014; Published 1 September 2014

Academic Editor: Engang Tian

Copyright (C) 2014 Hongjian Wang et al. This is an open access article distributed under the Creative Commons Attribution License, which permits unrestricted use, distribution, and reproduction in any medium, provided the original work is properly cited.

Considering the case of ASV (autonomous surface vehicle) navigating with low speed near water surface, a new method for design of roll motion controller is proposed in order to restrain wave disturbance effectively and improve roll stabilizing performance. Control system design is based on GPC (general predictive control) theory and working principle of zero-speed fin stabilizer. Coupling horizontal motion model of ASV is decoupled, and an equivalent transfer function of roll motion is obtained and transformed into a discrete difference equation through inverse Laplace transformation and Euler approximation. Finally, predictive model of GPC, namely, the difference equation of roll motion, is given. GPC algorithm of ASV roll motion is derived from performance index based on roll stabilizing performance and energy consumption used for driving fin stabilizer. In allusion to time-variant parameters in roll motion model, recursive least square method is adopted for parameter estimation. Simulation results of ASV roll motion control show better stabilizing performance and minimized energy consumption improved by self-adaptive GPC.

\section{Introduction}

ASV (autonomous surface vehicle) rolls severely if it is navigating near water surface and wave disturbance has an obvious effect on its motion attitude. Violent roll motion often discontinues normal working of ASV $[1,2]$. So, it is necessary to design an effective control pattern for solution to the problem of ASV motion attitude control. Moreover, it is hard for traditional fin stabilizer to generate enough lift when ASV is navigating with low speed. Consequently roll motion is very difficult to control at the moment. Then a new pattern of fin stabilizer working is required to realize effective roll control in low speed navigation. Marine Research Institute Netherlands, KoopNautic Holland, and Quantum Controls Ltd. have ever cooperated in the research on zero-speed fin stabilizer system [3]. Harbin Engineering University has designed and tested zero-speed fin stabilizers since 2005 [4]. Considering the characteristic of ASV motion with low speed near water surface, roll attitude is controlled by zero-speed fin stabilizer.
Roll attitude is often affected due to strong coupling among yaw, sway, and roll motion $[5,6]$. In the design of traditional roll stabilizing system, only roll motion is considered and coupling effect of yaw and sway on roll is neglected [7, 8]. So, control system based on ASV model with single degree of freedom usually cannot attain expected roll stabilizing performance in practical application. It is very hard to establish dynamic model of ASV because of uncertainty in motion model parameters especially for roll damp. There is a great error between theoretical value and actual value. Summing up the above, traditional PID control cannot adapt to parameter variation of ASV model and roll stabilizing performance is also affected as a result.

On the basis of some authentic references [9-11], coupling effect of yaw and sway on roll is considered and made as premise of issue discussion in this paper. GPC (general predictive control) is adopted in system design due to its well robustness for model identification error and uncertain time-lag or order of controlled system [12]. Compared to traditional control, strict requirement is not raised for model 


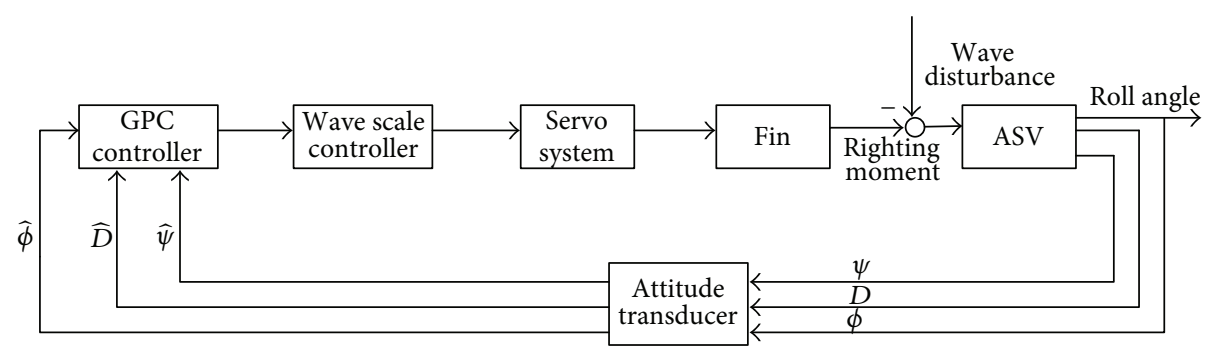

FIGURE 1: Scheme diagram of ASV roll stabilizing system.

structure. In the meantime, time variation, model mismatch, and disturbance uncertainty are also considered in GPC which is fit for roll motion control of ASV working near water surface. Zero-speed fin stabilizer would not generate enough lift force in low speed if it was in the working pattern of traditional fin stabilizer. So, zero-speed fin stabilizer works in another different pattern and usually generates lift force through revolving around its fin axis. Moment for driving fin stabilizer is large enough in order to satisfy roll stabilizing requirement. Consequently, energy consumption for roll attitude control is also very large. For small scale ASV, energy supplied to zero-speed fin stabilizer is often limited. So, it is necessary to reduce energy consumption for driving fin stabilizer, while roll stabilizing performance is satisfactory. Energy consumption and roll stabilizing performance are both considered in performance index of GPC in this paper. Finally, ASV roll attitude control and energy consumption saving are realized at the same time.

To sum up the above ideas, the assumption and associated limitation in this paper can be described as follows.

(a) ASV is navigating with low speed.

(b) Coupling effect of yaw and sway on roll is considered.

(c) Time variation of parameters in ASV motion model and disturbance uncertainty are considered in GPC.

\section{Roll Stabilizing Principle of ASV with Low Speed}

Schematic diagram of ASV roll stabilizing system is shown in Figure 1.

Roll attitude of ASV with low speed is controlled through actuation of system controller. Wings of fin stabilizer actively flap around fin axis with high frequency in sea water. Lift on the wing surface is generated under driving of servo system. Lift righting moment counteracts wave moment effectively, and then roll motion amplitude is reduced [13]. In Figure 1, $\psi, D$, and $\phi$ represent yaw angle, sway displacement, and roll angle, respectively. Similarly, $\widehat{\psi}, \widehat{D}$, and $\widehat{\phi}$ are measured values of corresponding variables. ASV model discussed in this paper is chosen from [14]. Relevant parameters of fin stabilizer are shown as follows: span length is $0.25 \mathrm{~m}$, chord length is $0.5 \mathrm{~m}$, and navigating speed is $1.832 \mathrm{~m} / \mathrm{s}$. According to roll stabilizing theory, lift values of traditional fin stabilizer are very small and several Newtons in quantity if fin size and navigating speed are set as values above. Lift force is too

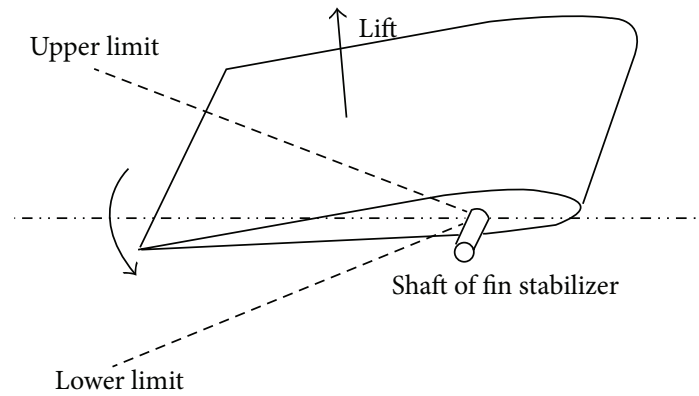

Figure 2: Principle of zero-speed fin stabilizer.

small to satisfy the need of roll stabilizing if traditional fin stabilizer is used under this condition. But conventional zerospeed fin stabilizer, which has the same size, can generate enough lift force to counteract wave disturbance in the case of low speed navigation near water surface. Thus, lift model of conventional zero-speed fin stabilizer [15] is adopted in the following discussion.

Principle of zero-speed fin stabilizer is shown in Figure 2. Considering special working pattern of zero-speed fin stabilizer, force analysis during normal working of fin commits to category of unsteady flow problem [16]. When zero-speed fin stabilizer flaps in perfect fluid or nonperfect fluid, lift generated on the fin can be analyzed by using potential theory and vortex action theory instead of fix wing theory. The forces on zero-speed fin stabilizer in unsteady flow are similar to tail fin of bionic fish [17]. The difference between them is that resistance produced by rotating is concerned for zerospeed fin stabilizer; however, thrust in the direction of going forward is concerned for bionic fish. The forces on tail fin of bionic fish are analyzed by many scholars. It is usually recognized that the forces can be divided into three types, namely, shape resistance, added mass force, and vortex force. The model of lift will be established by analyzing the forces in hydrodynamics. Through relevant deduction, lift model of zero-speed fin stabilizer [18] can be described as

$$
\begin{aligned}
L_{\text {zero }}= & {\left[\frac{e_{0} \rho}{3}\left(C_{d}+3 k\right)\left(3 a^{2} c+c^{3}\right) \omega^{2}+\frac{J_{I}}{d} \dot{\omega}\right] } \\
& \times \cos (\omega t)=\left(c_{1} \omega^{2}+c_{2} \dot{\omega}\right) \cos (\omega t) .
\end{aligned}
$$

In (1), $e_{0}$ is span length, $\rho$ is sea water density, $C_{d}$ is coefficient of drag force, $k$ is proportion factor, $2 a$ is chord 


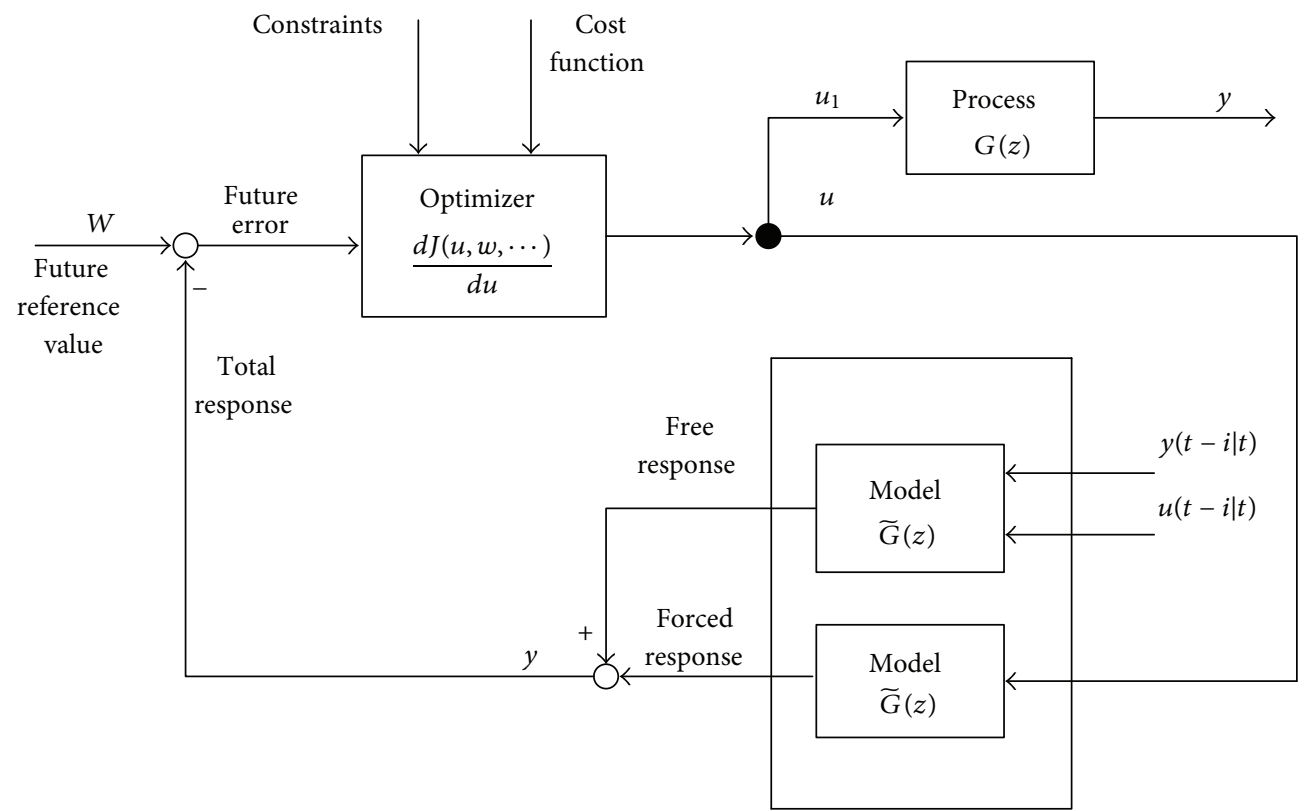

Figure 3: Basic structure of GPC.

length, $c$ is distance from fin axis to midpoint of chord length, $\omega$ is angular rate of fin wings flapping, $J_{I}$ is additional moment of inertia, $d$ is distance from fin axis to the point where force on additional mass acts, and $c_{1}, c_{2}$ are both constants.

Because the problem discussed in this paper is roll attitude control of ASV with low speed, it is necessary to consider additional effect of water flow on lift force while sea water flows through fin surface with relative flow speed. The additional lift is in relation to navigating speed, and it is timevariant; namely, additional lift can be denoted as $\Delta L_{\text {lift }}(V, t)$. Thus, lift model of fin stabilizer, when ASV is navigating with low speed, can be given by

$$
L_{\text {lift }}=L_{\text {zero }}+\Delta L_{\text {lift }}(V, t) .
$$

If navigating speed is $1.832 \mathrm{~m} / \mathrm{s}$, value of $\Delta L_{\text {lift }}(V, t)$ is much less than that of $L_{\text {zero }}$. Ratio of additional lift to total lift is $3 \%-4 \%$. So, (3) can be approximately accepted in simulations. Consider

$$
L_{\text {lift }} \approx L_{\text {zero }} .
$$

\section{Calculation of Wave Moment}

In research of ASV motion control, Pierson-Moskowitz spectrum with single parameter is often used [19] and its spectral density formula is given by

$$
S(\omega)=\frac{8.1 \times 10^{-3} g^{2}}{\omega^{5}} \exp \left[\frac{-3.11}{H_{s}^{2} \omega^{4}}\right] .
$$

Here, $S(\omega)$ is spectral density $\left(\mathrm{m}^{2} \cdot \mathrm{s}\right), \omega$ is wave frequency $(\mathrm{rad} / \mathrm{s})$, and $H_{s}$ is significant wave height (m). In the following simulation, spectral density $S(\omega)$ is divided into 30 wavebands in frequency domain, and each waveband is $\delta \omega$ in width.
Then, wave moment relevant to each component wave is added up, and total transient wave moment can be obtained and expressed as

$$
\begin{aligned}
& M_{\text {wave }}(t)= \sum_{i=1}^{30} M_{\text {wavei }}(t) \\
&=-\sum_{i=1}^{30} c_{M} l \nabla \rho(1-0.02 u \cos \gamma) \operatorname{sign}(\cos \gamma) f_{i} \\
& \quad \quad \times \cos \omega_{e i} t .
\end{aligned}
$$

Here, $f_{i}=a_{i}^{2} \omega_{i}^{2} \exp \left(-\omega_{i}^{2} h(t) / g\right), a_{i}=\left[2 S\left(\omega_{i}\right) \delta \omega\right]^{1 / 2}$, $h(t)$ is navigating depth, $\nabla$ is volumetric displacement, $u$ is navigating speed, $\gamma$ is heading angle, $c_{M}$ is a given coefficient of hydrodynamic force, $\rho$ is sea water density, $l$ is total length of AUV, $g$ is acceleration of gravity, $\omega_{e}$ is encountering frequency, and $\omega_{e}=-\omega-\left(\omega^{2} u / g\right) \cos \gamma$.

\section{ASV Motion Model}

Coupling horizontal motion model of ASV is considered in this paper. Detailed derivation of ASV motion model is specified in Appendix A.

\section{Design of Self-Adaptive GPC Controller}

5.1. Basic Structure of GPC. Basic structure of GPC is shown in Figure 3. GPC belongs to the group of "long-range predictive controllers" and generates a set of future control signals in each sampling interval, but only the first element of the control sequence is applied to the system input.

The prediction of the system output $\mathbf{y}$ is based on two different components. The "free response" represents the 
predicted behaviour of the output $\mathbf{y}(\mathbf{t}+\mathbf{j} \mid \mathbf{t}$ ) (in the range from $\mathbf{t}+\mathbf{1}$ to $\mathbf{t}+\mathbf{N})$, based on old outputs $\mathbf{y}(\mathbf{t}-\mathbf{i} \mid \mathbf{t})$ and inputs $\mathbf{u}(\mathbf{t}-\mathbf{i} \mid \mathbf{t})$, assuming a future control action of zero. The "forced response" represents the additional component of the output $y$ resulting from the optimisation criterion.

The total prediction is the sum of both components (for linear systems). Together with the known reference values, the future errors can be calculated by

$$
e(t+j t)=w(t+j t)-y(t+j t)
$$

with $\mathbf{j}$ counting from $\mathbf{1}$ to $\mathbf{N}$ (system stability will be improved if $\mathbf{N}$ increases, but response rapidity will deteriorate in the same time and vice versa. $\mathbf{N}$ can be quantified through combining stability with rapidity). Caused by these "future errors," future control signals are calculated to force the output to the desired reference values.

In addition to its well-known good control performance, the robustness properties make GPC interesting and realizable for practical control applications. For this purpose GPC offers a compact control strategy in terms of model mismatches, variable dead time, and disturbances.

5.2. GPC Algorithm. If wave disturbance is not taken into account, roll motion model of ASV can be expressed as

$$
A\left(z^{-1}\right) \Delta \phi(k)=B\left(z^{-1}\right) \Delta M_{\text {fin }}(k),
$$

where $A\left(z^{-1}\right)=1+a_{1} z^{-1}+a_{2} z^{-2}, B\left(z^{-1}\right)=b_{0}+b_{1} z^{-1}$, and $\Delta=1-z^{-1}$. Performance index of GPC is defined as

$$
\begin{aligned}
& J=E\left\{\sum_{j=N_{1}}^{N_{2}}\left(\phi(k+j)-\phi_{r}(k+j)\right)^{2}\right. \\
&\left.+\lambda \cdot \sum_{j=2}^{N_{2}+1} \Delta \alpha_{j} \cdot\left|M_{\text {fin }}(k+j-1)\right|\right\} .
\end{aligned}
$$

In (8), E represents operation of calculating mathematical expectation, $N_{1} N_{2}$ are minimal time domain and maximal time domain of GPC prediction, respectively, $\phi$ denotes roll angle, $\phi_{r}(k+j)$ is expected value of roll angle at the time $k+j$, and $\lambda$ is weight coefficient of performance index. $\Delta M_{\text {fin }}(k+$ $j)=0, j=N_{u}, \ldots, N_{2}$, where $N_{u}$ is control time domain, which means control input $M_{\text {fin }}(k)$ keeps invariant after $N_{u}$ steps. $\Delta \alpha_{j}$ is variation of fin angle. Consider

$$
\alpha_{k+j}=\alpha_{k+j-1}+\omega_{k+j}^{\prime} \cdot T,
$$

where $\alpha_{k+j} \alpha_{k+j-1}$ are fin angles at the time $k+j$ and $k+j-$ 1 , respectively, $\omega_{k+j}^{\prime}$ is angular rate of fin wings flapping, and $T$ is sampling period. Considering frequent flapping of zerospeed fin stabilizer wings, $T$ is set as $1 \mathrm{~ms}$. From (9), variation of fin angle can be calculated as

$$
\Delta \alpha_{j}=\alpha_{k+j}-\alpha_{k+j-1}=\omega_{k+j}^{\prime} \cdot T
$$

In order to obtain optimal predictive value of $\phi(k+j)$, the following Diophantine equations are considered firstly:

$$
\begin{gathered}
1=E_{j}\left(z^{-1}\right) A\left(z^{-1}\right) \Delta+z^{-j} F_{j}\left(z^{-1}\right) \\
E_{j}\left(z^{-1}\right) B\left(z^{-1}\right)=G_{j}\left(z^{-1}\right)+z^{-j} H_{j}\left(z^{-1}\right),
\end{gathered}
$$

where $j=1, \ldots, N_{2}, E_{j}=e_{0}+e_{1} z^{-1}+\cdots+e_{j-1} z^{-j+1}$, $F_{j}=f_{0}^{j}+f_{1}^{j} z^{-1}+f_{2}^{j} z^{-2}, G_{j}=g_{0}+g_{1} z^{-1}+\cdots+g_{j-1} z^{-j+1}$, and $H_{j}=h_{0}^{j}$. Through deduction from (7) and (11), optimal predictive value of $\phi(k+j)$ is obtained and given by

$$
\phi(k+j)=G_{j} \Delta M_{\mathrm{fin}}(k+j)+F_{j} \phi(k)+H_{j} \Delta M_{\mathrm{fin}}(k),
$$

where $j=1, \ldots, N_{2}$. Equivalent matrix form of (12) is described as

$$
\phi=G \Delta M_{\text {fin }}+F \phi(k)+H \Delta M_{\text {fin }}(k),
$$

where

$$
\begin{aligned}
& \phi^{T}=\left[\phi(k+1), \ldots, \phi\left(k+N_{2}\right)\right], \\
& M_{\text {fin }}^{T}=\left[M_{\text {fin }}(k+1), \ldots, M_{\text {fin }}\left(k+N_{2}\right)\right] \text {, } \\
& F^{T}=\left[F_{1}, \ldots, F_{N_{2}}\right] \text {, } \\
& H^{T}=\left[H_{1}, \ldots, H_{N_{2}}\right] \text {, } \\
& G=\left[\begin{array}{cccccc}
g_{0} & & & & & \\
g_{1} & g_{0} & & & & \\
\vdots & \vdots & \ddots & & & \\
g_{N_{u}-1} & g_{N_{u}-2} & \cdots & g_{0} & & \\
\vdots & \vdots & \vdots & \vdots & \ddots & \\
g_{N_{2}-1} & g_{N_{2}-2} & \cdots & g_{N_{2}-N_{u}} & \cdots & g_{0}
\end{array}\right]
\end{aligned}
$$

If $\phi_{r}^{T}=\left[\phi_{r}(k+1), \ldots, \phi_{r}\left(k+N_{2}\right)\right]$ and $\Delta \alpha=\left[\omega_{k+2}^{\prime}\right.$. $\left.T, \ldots, \omega_{k+N_{2}+1}^{\prime} \cdot T\right],(8)$ can be expressed as the following matrix form:

$$
J=E\left\{\left(\phi-\phi_{r}\right)^{T}\left(\phi-\phi_{r}\right)+\lambda \cdot \Delta \alpha \cdot \operatorname{abs}\left(M_{\text {fin }}\right)\right\},
$$

where $\operatorname{abs}(\cdot)$ denotes the matrix composed of absolute values of original matrix elements and $\lambda$ is selected according to the range of roll angle and corresponding energy consumption used for roll stabilizing. For simplification of the following discussion, $N_{u}=1$, then

$$
\begin{aligned}
\operatorname{abs}\left(M_{\text {fin }}\right)= & \operatorname{sign}\left[M_{\text {fin }}(k+1)\right] \\
& \cdot M_{\text {fin }}(k+1) \cdot[1,1, \ldots, 1]^{T} .
\end{aligned}
$$


In (16), sign $(\cdot)$ denotes signum function. Equations (13) and (16) are substituted into (15), and (17) is obtained. Consider

$$
\left.\cdot[1,1, \ldots, 1]^{T}+(\Delta \alpha)^{T} \cdot \operatorname{sign}\left[M_{\text {fin }}(k+1)\right]\right\}
$$

$$
\begin{aligned}
2 \Delta G^{T} & {\left[G \Delta M_{\mathrm{fin}}+F \phi(k)+H \Delta M_{\mathrm{fin}}(k)-\phi_{r}\right] } \\
& +\lambda \cdot\left\{\frac{\partial(\Delta \alpha)}{\partial M_{\mathrm{fin}}} \cdot \operatorname{sign}\left[M_{\mathrm{fin}}(k+1)\right] \cdot M_{\mathrm{fin}}(k+1)\right.
\end{aligned}
$$$$
=0 \text {. }
$$

In (17),

$$
\begin{gathered}
\frac{\partial(\Delta \alpha)}{\partial M_{\mathrm{fin}}}=\left[\begin{array}{cccc}
\frac{\partial \omega_{k+2}^{\prime}}{\partial M_{\mathrm{fin}}(k+1)} & \frac{\partial \omega_{k+3}^{\prime}}{\partial M_{\mathrm{fin}}(k+1)} & \cdots & \frac{\partial \omega_{k+N_{2}+1}^{\prime}}{\partial M_{\mathrm{fin}}(k+1)} \\
\vdots & \vdots & \vdots & \vdots \\
\frac{\partial \omega_{k+2}^{\prime}}{\partial M_{\mathrm{fin}}\left(k+N_{2}\right)} & \frac{\partial \omega_{k+3}^{\prime}}{\partial M_{\mathrm{fin}}\left(k+N_{2}\right)} & \cdots & \frac{\partial \omega_{k+N_{2}+1}^{\prime}}{\partial M_{\mathrm{fin}}\left(k+N_{2}\right)}
\end{array}\right] \cdot T \\
\Delta M_{\mathrm{fin}}=\left[\begin{array}{ll}
\Delta M_{\mathrm{fin}}(k+1), 0, \ldots, 0
\end{array}\right]^{T}
\end{gathered}
$$

If

$$
G^{\prime}=G^{T} G=\left[\begin{array}{cccc}
g_{11}^{\prime} & g_{12}^{\prime} & \cdots & g_{1 N_{2}}^{\prime} \\
\vdots & \vdots & \vdots & \vdots \\
g_{N_{2} 1}^{\prime} & g_{N_{2} 2}^{\prime} & \cdots & g_{N_{2} N_{2}}^{\prime}
\end{array}\right]
$$

and $p^{T}=[1,0, \ldots, 0],(17)$ is multiplied by $p^{T}$. Through further simplification, (20) is obtained. Consider

$$
\begin{aligned}
\left\{2 \Delta p^{T} G^{\prime} \Delta+\lambda \cdot p^{T} \cdot \frac{\partial(\Delta \alpha)}{\partial M_{\text {fin }}} \cdot \operatorname{sign}\left[M_{\text {fin }}(k+1)\right]\right\} \cdot M_{f i n} \\
=2 \Delta p^{T} G^{T}\left[\phi_{r}-F \phi(k)-H \Delta M_{\text {fin }}(k)\right] \\
\quad-\lambda \cdot p^{T} \cdot(\Delta \alpha)^{T} \cdot \operatorname{sign}\left[M_{\text {fin }}(k+1)\right] .
\end{aligned}
$$

Since $M_{\mathrm{fin}}(k+1)=2 \cdot L_{\mathrm{lift}} \cdot l_{f}=2 \cdot l_{f} \cdot\left(c_{1} \omega_{k+1}^{\prime}{ }^{2}+c_{2} \omega_{k+1}^{i}\right)$. $\cos \alpha_{k+1}, \omega_{k+2}^{\prime}=\omega_{k+1}^{\prime}+\omega_{k+1}^{i} \cdot T$, partial derivative of $\omega^{\prime}$ with respect to $M_{\text {fin }}$ is given by

$$
\begin{gathered}
\frac{\partial \omega_{k+2}^{\prime}}{\partial M_{\text {fin }}(k+1)}=\frac{\partial \omega_{k+1}^{i}}{\partial M_{\text {fin }}(k+1)} \cdot T \\
=\frac{T}{2 \cdot l_{f} \cdot c_{2} \cdot \cos \alpha_{k+1}} \\
\frac{\partial \omega_{k+3}^{\prime}}{\partial M_{\text {fin }}(k+1)} \\
=\frac{\partial\left(\omega_{k+2}^{\prime}+\omega_{k+2}^{i} \cdot T\right)}{\partial M_{\text {fin }}(k+1)}=\frac{\partial \omega_{k+2}^{\prime}}{\partial M_{\text {fin }}(k+1)} \\
=\frac{T}{2 \cdot l_{f} \cdot c_{2} \cdot \cos \alpha_{k+1}}
\end{gathered}
$$

$$
\begin{aligned}
& \frac{\partial \omega_{k+4}^{\prime}}{\partial M_{\mathrm{fin}}(k+1)} \\
& =\frac{\partial\left(\omega_{k+3}^{\prime}+\omega_{k+3}^{i} \cdot T\right)}{\partial M_{\mathrm{fin}}(k+1)}=\frac{\partial \omega_{k+3}^{\prime}}{\partial M_{\mathrm{fin}}(k+1)} \\
& =\frac{T}{2 \cdot l_{f} \cdot c_{2} \cdot \cos \alpha_{k+1}}
\end{aligned}
$$

$$
\begin{aligned}
\frac{\partial \omega_{k+N_{2}+1}^{\prime}}{\partial M_{\mathrm{fin}}(k+1)} & =\frac{\partial\left(\omega_{k+N_{2}}^{\prime}+\omega_{k+N_{2}}^{\prime} \cdot T\right)}{\partial M_{\mathrm{fin}}(k+1)} \\
& =\frac{\partial \omega_{k+N_{2}}^{\prime}}{\partial M_{\mathrm{fin}}(k+1)} \\
& =\frac{T}{2 \cdot l_{f} \cdot c_{2} \cdot \cos \alpha_{k+1}},
\end{aligned}
$$

where $l_{f}$ is lever of righting moment. So, in (20),

$$
\begin{aligned}
p^{T} & \cdot \frac{\partial(\Delta \alpha)}{\partial M_{\text {fin }}} \\
= & {\left[\frac{\partial \omega_{k+2}^{\prime}}{\partial M_{\text {fin }}(k+1)} \frac{\partial \omega_{k+3}^{\prime}}{\partial M_{\text {fin }}(k+1)} \cdots \frac{\partial \omega_{k+N_{2}+1}^{\prime}}{\partial M_{\text {fin }}(k+1)}\right] } \\
& \cdot T \\
= & \frac{T^{2}}{2 \cdot l_{f} \cdot c_{2} \cdot \cos \alpha_{k+1}} \cdot\left[\begin{array}{llll}
1 & 1 & \cdots & 1
\end{array}\right] \\
p^{T} \cdot(\Delta \alpha)^{T} & \\
= & \omega_{k+2}^{\prime} \cdot T
\end{aligned}
$$




$$
\begin{aligned}
& =\left(\omega_{k+1}^{\prime}+\omega_{k+1}^{i} \cdot T\right) \cdot T \\
& =\omega_{k+1}^{\prime} \cdot T+\omega_{k+1}^{i} \cdot T^{2} \\
& =\omega_{k+1}^{\prime} \cdot T+T^{2} \cdot \frac{M_{\mathrm{fin}}(k+1)-2 l_{f} \mathcal{c}_{1} \omega_{k+1}^{\prime}{ }^{2} \cos \alpha_{k+1}}{2 l_{f} \mathcal{c}_{2} \cos \alpha_{k+1}} .
\end{aligned}
$$

Considering $\phi_{r}=\left[\phi_{r}(k+1), \ldots, \phi_{r}\left(k+N_{2}\right)\right]^{T}=$ $[0, \ldots, 0]^{T},(22)$ are substituted into $(20)$; then

$$
\begin{aligned}
& \left\{\frac{\lambda\left(N_{2}+1\right) T^{2}}{2 l_{f} \mathcal{c}_{2} \cos \alpha_{k+1}} \cdot \operatorname{sign}\left[M_{\mathrm{fin}}(k+1)\right]\right. \\
& \left.+2\left(g_{11}^{\prime}+g_{12}^{\prime}+\cdots+g_{1 N_{2}}^{\prime}\right)\right\} \\
& \cdot M_{\text {fin }}(k+1) \\
& =\left[4\left(g_{11}^{\prime}+g_{12}^{\prime}+\cdots+g_{1 N_{2}}^{\prime}\right)\right. \\
& \left.-2\left(g_{0} H_{1}+g_{1} H_{2}+\cdots+g_{N_{2}-1} H_{N_{2}}\right)\right] \\
& \cdot M_{\text {fin }}(k) \\
& +\left[4\left(g_{0} H_{1}+g_{1} H_{2}+\cdots+g_{N_{2}-1} H_{N_{2}}\right)\right. \\
& \left.-2\left(g_{11}^{\prime}+g_{12}^{\prime}+\cdots+g_{1 N_{2}}^{\prime}\right)\right] \\
& \cdot M_{\text {fin }}(k-1) \\
& -2\left(g_{0} H_{1}+g_{1} H_{2}+\cdots+g_{N_{2}-1} H_{N_{2}}\right) \cdot M_{\text {fin }}(k-2) \\
& -2\left(g_{0} F_{1}+g_{1} F_{2}+\cdots+g_{N_{2}-1} F_{N_{2}}\right) \cdot \phi(k) \\
& +2\left(g_{0} F_{1}+g_{1} F_{2}+\cdots+g_{N_{2}-1} F_{N_{2}}\right) \cdot \phi(k-1) \\
& -\lambda \cdot \operatorname{sign}\left[M_{\mathrm{fin}}(k+1)\right] \cdot\left(\omega_{k+1}^{\prime} \cdot T-\frac{c_{1}}{c_{2}} \omega_{k+1}^{\prime}{ }^{2} T^{2}\right) \text {. }
\end{aligned}
$$

Equation (23) is abbreviated as (24), where $A_{1}, A_{2}, A_{3}, A_{4}$, $B_{1}\left(z^{-1}\right), B_{2}\left(z^{-1}\right), C$ are coefficients of corresponding terms in (23). Consider

$$
\begin{aligned}
A_{1} \cdot M_{\text {fin }}(k+1) & \\
= & A_{2} \cdot M_{\text {fin }}(k)+A_{3} \cdot M_{\text {fin }}(k-1)+A_{4} \cdot M_{\text {fin }}(k-2) \\
& \quad+B_{1}\left(z^{-1}\right) \cdot \phi(k)+B_{2}\left(z^{-1}\right) \cdot \phi(k-1)+C .
\end{aligned}
$$

So, GPC algorithm can be described as

$$
\begin{aligned}
& M_{\text {fin }}(k+1) \\
& =\left(\left[A_{2} \cdot M_{\text {fin }}(k)+A_{3} \cdot M_{\text {fin }}(k-1)+A_{4} \cdot M_{\text {fin }}(k-2)\right.\right. \\
& \left.\left.\quad+B_{1}\left(z^{-1}\right) \cdot \phi(k)+B_{2}\left(z^{-1}\right) \cdot \phi(k-1)+C\right]\right) \\
& \quad \times\left(A_{1}\right)^{-1} .
\end{aligned}
$$

5.3. Recursive Solution to Diophantine Equations. GPC algorithm obtained in Section 5.2 is followed by some relevant conclusions; namely, in Diophantine equations (9)-(10), $E_{j}\left(z^{-1}\right), F_{j}\left(z^{-1}\right), G_{j}\left(z^{-1}\right)$, and $H_{j}\left(z^{-1}\right)$ vary with predictive step number $j$ and need recalculation. In order to save calculation time and use the information in step backward, $E_{j}\left(z^{-1}\right), F_{j}\left(z^{-1}\right), G_{j}\left(z^{-1}\right)$, and $H_{j}\left(z^{-1}\right)$ are calculated with recursive method. Through derivation, recursive formulas of coefficients in Diophantine equations are obtained and listed in Appendix B.

5.4. Parameter Estimation of ASV Roll Motion Model. Parameter estimation is necessary due to time-variant parameters in ASV roll motion model. Recursive least square method is used here and algorithm for estimating parameter vector is given in Appendix C.

\subsection{Steps of Self-Adaptive GPC Algorithm}

(1) Assign values to $N_{1}, N_{2}, \lambda$, and $\mu$.

(2) Set initial vector $\theta(0)$ and initial matrix $\Omega(0)$. ( $\Omega(0)$ is a positive definite matrix.)

(3) Measure current roll angle $\phi(k)$ and fetch corresponding set value $\phi_{r}$.

(4) Estimate parameter vector $\theta(k)$ with recursive least square method and calculate coefficients of corresponding terms in $A\left(z^{-1}\right)$ and $B\left(z^{-1}\right)$, namely, $a_{1}, a_{2}, b_{0}$, and $b_{1}$.

(5) Calculate $E_{j}\left(z^{-1}\right), F_{j}\left(z^{-1}\right), G_{j}\left(z^{-1}\right)$, and $H_{j}\left(z^{-1}\right)$ according to (B.1).

(6) Substitute $E_{j}\left(z^{-1}\right), F_{j}\left(z^{-1}\right), G_{j}\left(z^{-1}\right)$, and $H_{j}\left(z^{-1}\right)$ into (25) and then calculate optimal control variable $M_{\text {fin }}(k+1)$.

(7) Exert $M_{\text {fin }}(k+1)$ on current roll motion model of ASV.

(8) Return to step (3).

\section{Results}

Total length of ASV is $5.3 \mathrm{~m}$, ASV height is $0.5 \mathrm{~m}$, ASV width is $1 \mathrm{~m}$, and navigating speed is $1.832 \mathrm{~m} / \mathrm{s}$. Distance from fin axis to midpoint of chord length is $0.125 \mathrm{~m}$, and maximum of fin angle is $60^{\circ}$. Parameters of self-adaptive GPC algorithm are set as $N_{1}=1, N_{2}=5, N_{u}=1, \lambda=3 \times 10^{-7}$, and $\mu=0.5$. Initial values for parameter estimation are $\theta(0)=\left[-2.5947,2.2035,-0.6088,1.1152 \times 10^{-4},-2.1877 \times\right.$ 


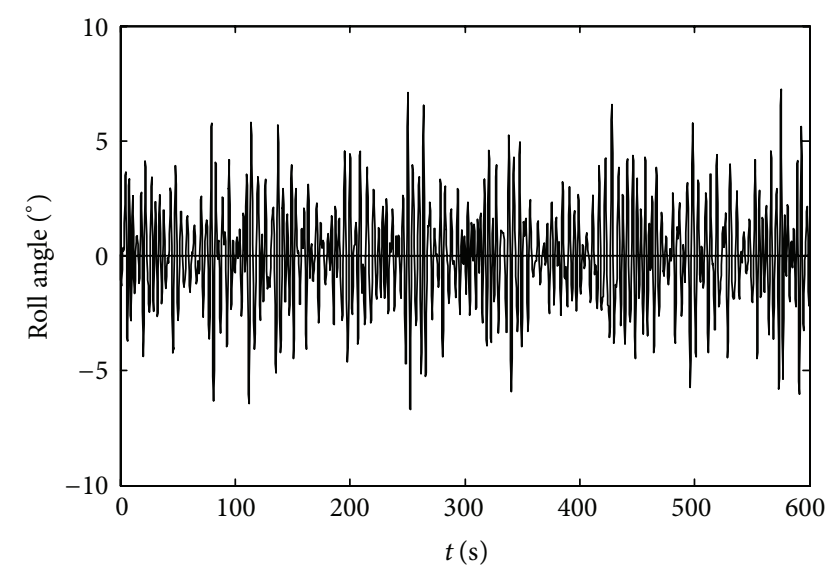

FIGURE 4: Simulation without roll reduction when $H_{s}=1$ and $\gamma=$ $45^{\circ}$.

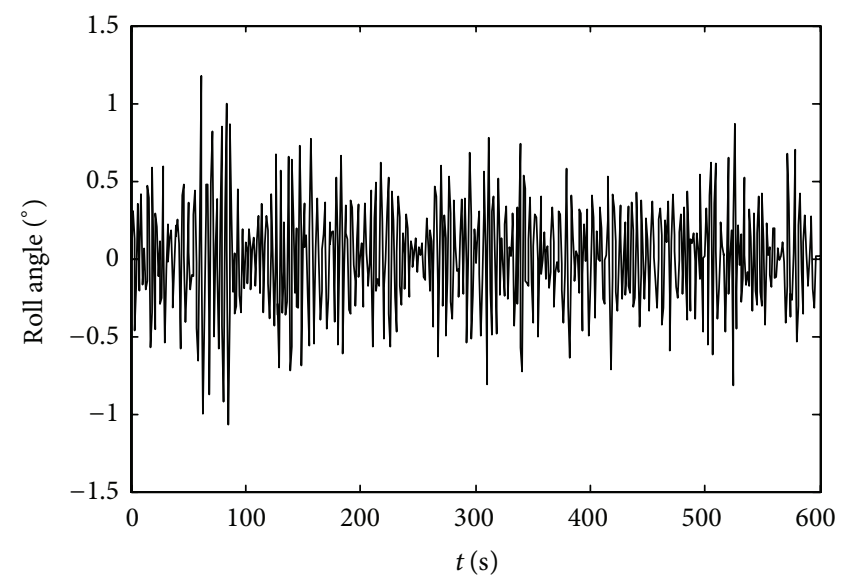

FIGURE 5: Simulation of self-adaptive GPC when $H_{s}=1$ and $\gamma=45^{\circ}$.

$10^{-4}, 1.0725 \times 10^{-4}$ ] and $\Omega(0)=I_{6 \times 6}$ (identity matrix with 6 dimensions). Set values of roll angle are all 0 ; namely, $\phi_{r}(k+$ $j)=0(j=1,2,3,4,5)$. Significant wave height and heading angle are denoted by $H_{s}$ and $\gamma$, respectively. Simulation results under different sea conditions are shown in Figures 4, 5, 6, 7, $8,9,10,11$, and 12 .

Roll stabilizing performance in Table 1 and energy-saving ratio in Table 2 are calculated according to the following formulas, respectively:

RSP

$$
\begin{gathered}
=\frac{\text { SDRA without roll control }- \text { SDRA with GPC }}{\text { SDRA without roll control }} \times 100 \% \\
\text { ESR }=\frac{\text { EC based on } J_{0}-\text { EC based on } J}{\text { EC based on } J_{0}} \times 100 \% .
\end{gathered}
$$

Meanings for RSP, SDRA, ESR, and EC are shown as follows:

RSP: roll stabilizing performance,

SDRA: standard deviation of roll angle,

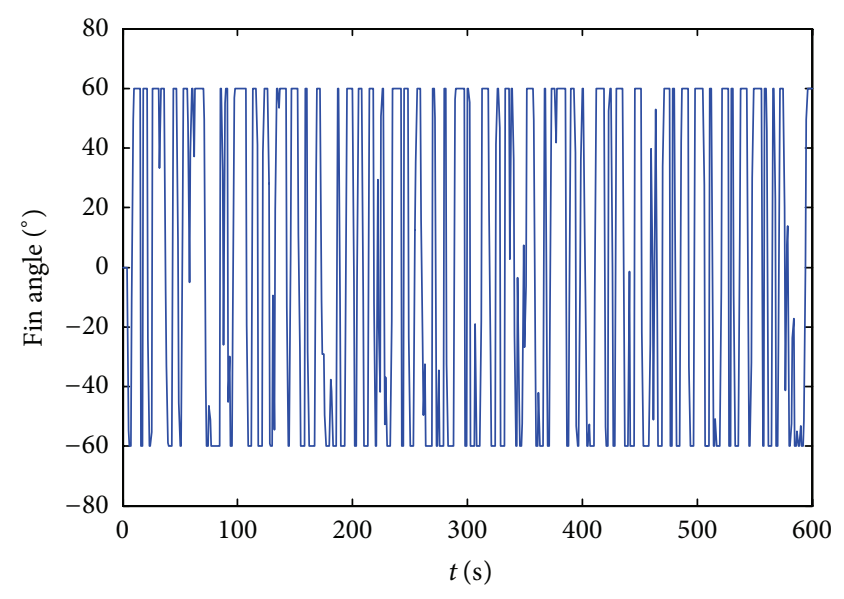

FIGURE 6: Fin angle of self-adaptive GPC when $H_{s}=1$ and $\gamma=45^{\circ}$.

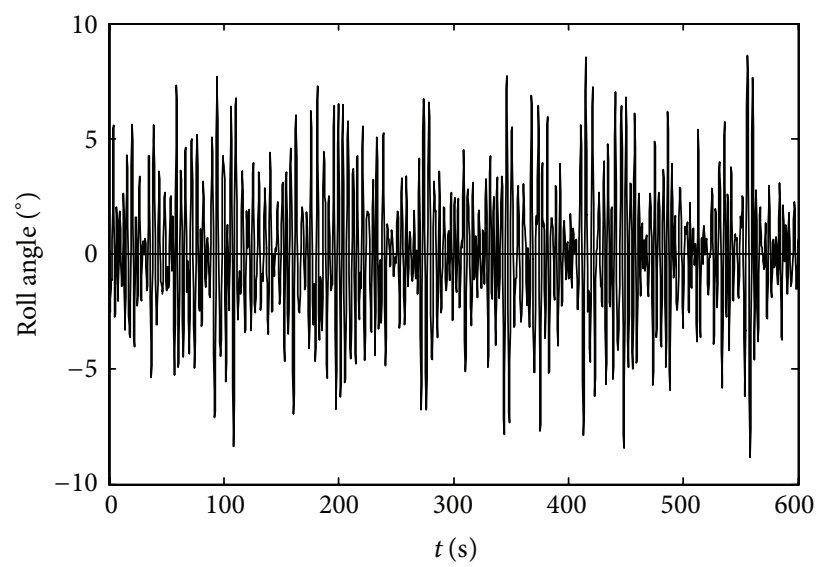

FIGURE 7: Simulation without roll reduction when $H_{s}=1$ and $\gamma=$ $90^{\circ}$.

\section{ESR: energy-saving ratio, \\ EC: energy consumption.}

According to [20,21], expression of $J$ is given by (8). In order to demonstrate the improved performance of roll stabilizing system based on energy optimization design, $J_{0}$ is defined as the performance of GPC only considering roll stabilizing effectiveness; namely,

$$
J_{0}=E\left\{\sum_{j=1}^{5}\left(\phi(k+j)-\phi_{r}(k+j)\right)^{2}\right\},
$$

where $\phi$ denotes roll angle, $\phi_{r}(k+j)$ is expected value of roll angle at the time $k+j$, and $E$ represents operation of calculating mathematical expectation.

Energy consumption is calculated according to the following formula:

$$
\mathrm{EC}=\sum_{k=1}^{600}\left|M_{\mathrm{fin}}(k)\right| \Delta \alpha_{k}
$$




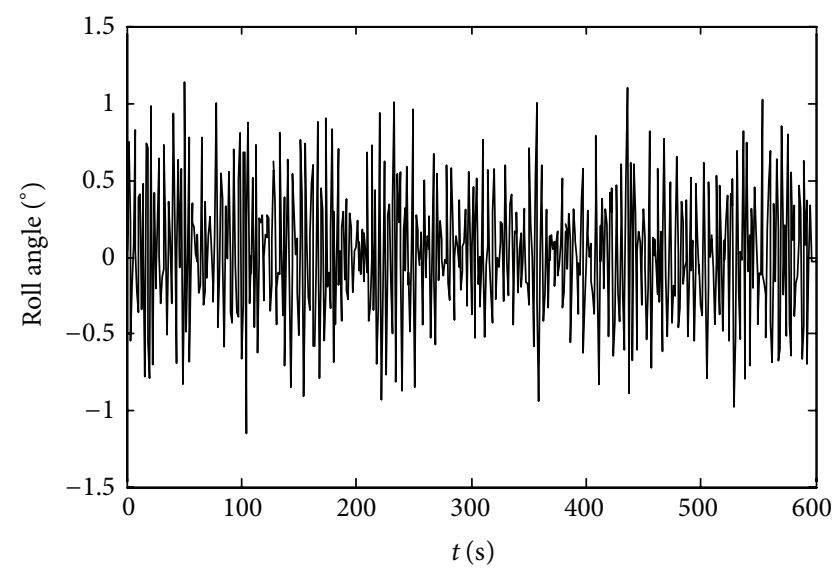

FIGURE 8: Simulation of self-adaptive GPC when $H_{s}=1$ and $\gamma=90^{\circ}$.

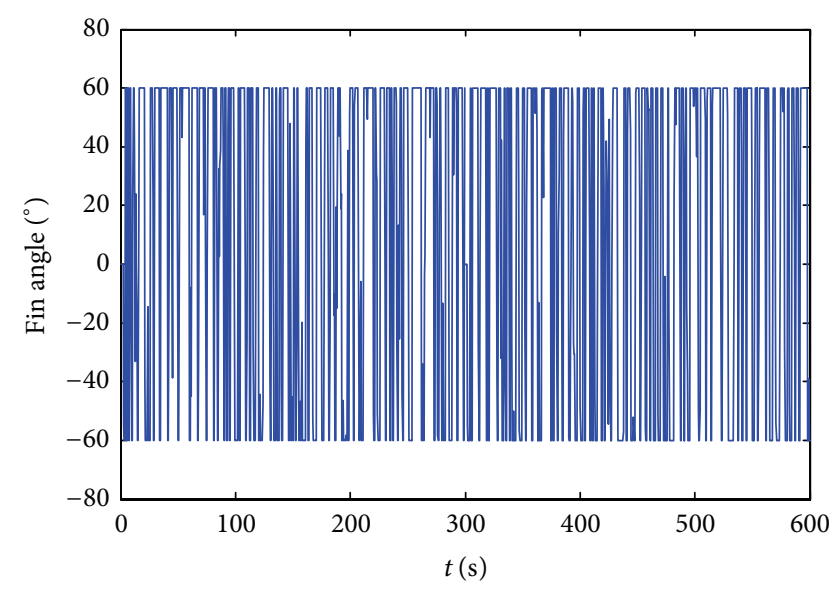

FIgURE 9: Fin angle of self-adaptive GPC when $H_{s}=1$ and $\gamma=90^{\circ}$.

where $M_{\text {fin }}$ is righting moment generated by zero-speed fin stabilizer and $\Delta \alpha_{k}$ is variation of fin angle.

In order to demonstrate the effectiveness of saving energy used for roll stabilizing, energy consumption based on $J$ is compared with energy consumption based on $J_{0}$ as shown in Figure 13. Simulation results shown in Figures 4-12 demonstrate that self-adaptive GPC embodies favorable robustness and satisfactory performance of roll stabilizing. There is no instability phenomenon in ASV roll motion. Conventional zero-speed fin stabilizer is used for roll stabilizing of ASV navigating with low speed near water surface. Roll stabilizing performance is favorable as shown in Table 1. Calculation results of energy consumption in Table 2 demonstrate that energy consumption based on performance index $J$ is less than energy consumption based on performance index $J_{0}$. In Figure 13, curves of energy consumption versus heading angle are displayed and corresponding maximums in energy consumption both occur in $90^{\circ}$. The heading angle increases with a step of $20^{\circ}$ in Figure 13. The two curves based on $J_{0}$ and $J$ obey normal distribution approximately, since motion attitude of ASV is obviously dominated by roll when heading angle varies in $75^{\circ}-105^{\circ}$ and is mainly affected by pitch and heave in $0^{\circ}-15^{\circ}$ or $165^{\circ}-180^{\circ}$. For the same reason, frequency

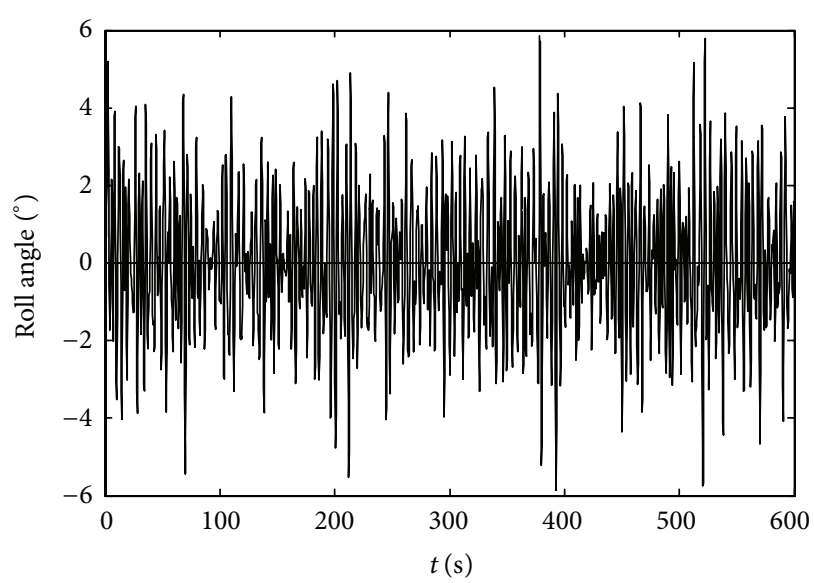

FIGURE 10: Simulation without roll reduction when $H_{s}=1$ and $\gamma=$ $135^{\circ}$.

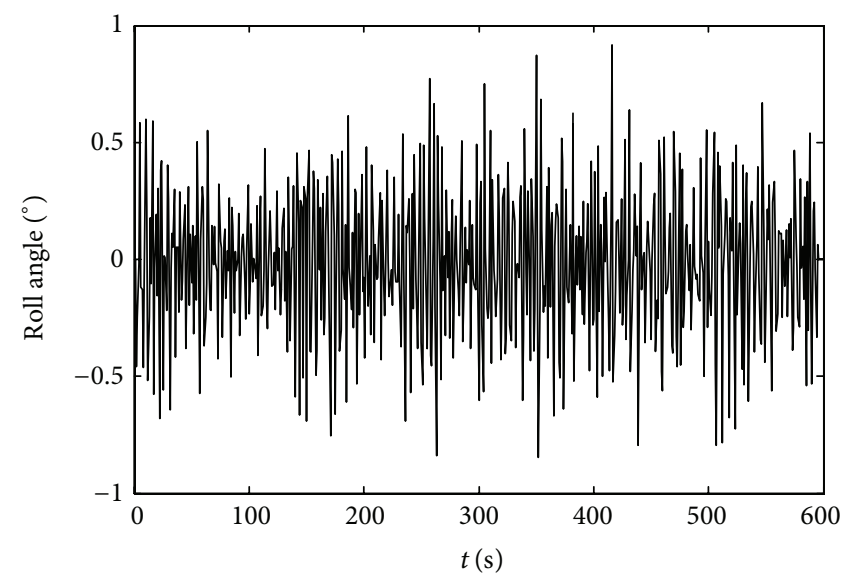

FIGURE 11: Simulation of self-adaptive GPC when $H_{s}=1$ and $\gamma=$ $135^{\circ}$.

of fin angle differs in Figures 6, 9, and 12; namely, fin flaps more frequently when $\gamma=90^{\circ}$ than $\gamma=45^{\circ}$ and $135^{\circ}$. Since control of $M_{\text {fin }}$ is implemented through adjustment of $\dot{\omega}^{\prime}$ and $\omega^{\prime}$ with regard to zero-speed fin stabilizer, which is different from fin angle control of traditional fin stabilizer, shortterm saturation occurs in the maximal fin angle as shown in Figures 6, 9, and 12 when $\omega^{\prime}$ is equal to zero. Figure 13 adequately shows the effectiveness of saving energy used for roll stabilizing. Satisfactory performance of roll stabilizing is also shown in Figures 14-15 and Figures 17-18, which correspond to different significant wave heights $\left(H_{s}=1.2 \mathrm{~m}\right.$ and $1.5 \mathrm{~m}$ ) when $\gamma=90^{\circ}$. Figures 16 and 19 show that the method proposed in this paper is effective on saving energy used for roll stabilizing when sea condition varies.

Through similar derivation, self-adaptive GPC is applied in traditional design of roll stabilizing system, where effects of sway and yaw on roll are disregarded. At this time, roll motion model is given by (D.4) in Appendix D and performance index of GPC is still given by (8). Detailed derivation of control law is not given here. Figures 20 and 21 show the roll stabilizing performances of traditional design. Compared 


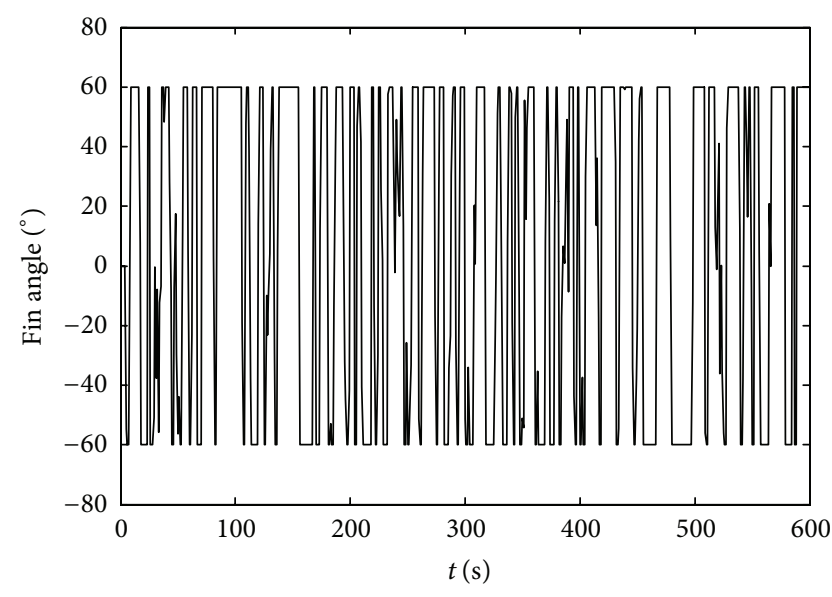

FIGURE 12: Fin angle of self-adaptive GPC when $H_{s}=1$ and $\gamma=$ $135^{\circ}$.

to Figures 15 and 18, roll stabilizing performances in Figures 20 and 21 are not quite satisfied. This result proves that the method in this paper is obviously superior to traditional design method, since roll motion model is more accurate if coupling effects are considered adequately.

In order to prove robustness of self-adaptive GPC controller designed in this paper, simulation experiment is conducted when significant wave height $H_{s}$ is $1 \mathrm{~m}$ and wave encounter angle $\gamma=90 \mathrm{deg}$. Uncertain parameters in ASV motion model (A.8) are described as $\Delta \overline{a_{1}}=0.08[1+$ $\sin (0.2 t)] \overline{a_{1}}, \Delta \overline{a_{2}}=0.08[1+\sin (0.2 t)] \overline{a_{2}}, \Delta \overline{b_{1}}=0.1[1+$ $\sin (0.2 t)] \overline{b_{1}}$, and $\Delta \overline{b_{2}}=0.1[1+\sin (0.2 t)] \overline{b_{2}}$, where $\overline{a_{1}}, \overline{a_{2}}, \overline{b_{1}}$, and $\overline{b_{2}}$ are corresponding normalized values of hydrodynamic force coefficients, and $\Delta \overline{a_{1}}, \Delta \overline{a_{2}}, \Delta \overline{b_{1}}$, and $\Delta \overline{b_{2}}$ represent their corresponding uncertain sections. Figure 22 shows the curve of roll angle without roll control under above uncertainty conditions, and Figure 23 shows corresponding curve with self-adaptive GPC. Working process of fin stabilizer is also described in Figure 24. Simulation results prove that selfadaptive GPC can be used to avoid parameter uncertainty in ASV motion model. Favorable stability and robustness demonstrate that the designed controller is effective when wave disturbance and ASV parameters are not determined.

Figures 25, 26, and 27 show the simulation results when ASV is navigating under rough sea conditions that significant wave height $\left(H_{s}\right)$ is $3 \mathrm{~m}$. As shown in Figure 25, the curve of roll angle violently varies between $-25^{\circ}$ and $25^{\circ}$ when ASV roll motion is not controlled. If self-adaptive GPC is adopted for roll reduction, the range of roll angle is reduced to $\left(-6^{\circ}\right.$, $6^{\circ}$ ). Roll stabilizing performance is satisfied, and the ability to cope with rough sea conditions proves the robustness of self-adaptive GPC. Simulation results demonstrate that selfadaptive GPC proposed in this paper is an effective method for the controller design of ASV roll motion, especially under rough sea conditions.

\section{Conclusions}

In the end, conclusions are drawn from analysis of simulation results; namely, ASV roll stabilizing performance is favorable under self-adaptive GPC, energy consumption can be reduced, and utilization rate of energy resource is raised by means of optimization if energy consumption is considered in performance index of GPC.

\section{Appendices}

\section{A. Decoupling and Discretization of ASV Motion Model}

Coupling horizontal motion model discussed in this paper is obtained by neglecting relevant parameters of ASV vertical motion (heave and pitch) and introducing the term of wave moment into roll equation. ASV coupling horizontal motion model is expressed as

$$
\begin{aligned}
{\left[\begin{array}{lll}
a_{11} & a_{12} & a_{13} \\
a_{21} & a_{22} & a_{23} \\
a_{31} & a_{32} & a_{33}
\end{array}\right] \cdot\left[\begin{array}{l}
\dot{v} \\
\dot{p} \\
\dot{r}
\end{array}\right]=} & V \cdot\left[\begin{array}{lll}
b_{11} & b_{12} & b_{13} \\
b_{21} & b_{22} & b_{23} \\
b_{31} & b_{32} & b_{33}
\end{array}\right] \\
& \cdot\left[\begin{array}{c}
v \\
p \\
r
\end{array}\right]+\left[\begin{array}{c}
0 \\
f+M_{\text {wave }}+M_{\text {fin }} \\
0
\end{array}\right],
\end{aligned}
$$

where $a_{11}=m-(1 / 2) \rho l^{3} Y_{\dot{v}}^{\prime}, a_{12}=-\left(m z_{G}+(1 / 2) \rho l^{4} Y_{\dot{p}}^{\prime}\right)$, $a_{13}=-(1 / 2) \rho l^{4} Y_{\dot{r}}^{\prime}, a_{21}=m z_{G}-(1 / 2) \rho l^{4} K_{\dot{v}}^{\prime}, a_{22}=I_{x}-$ $(1 / 2) \rho l^{5} K_{\dot{p}}^{\prime}, a_{23}=-(1 / 2) \rho l^{5} K_{\dot{r}}^{\prime}, a_{31}=-(1 / 2) \rho l^{4} N_{\dot{v}}^{\prime}, a_{32}=$ $-\left(I_{x z}+(1 / 2) \rho l^{5} N_{\dot{p}}^{\prime}\right), a_{33}=I_{z}-(1 / 2) \rho l^{5} N_{\dot{r}}^{\prime}, b_{11}=(1 / 2) \rho l^{2} Y_{v}^{\prime}$, $b_{12}=(1 / 2) \rho l^{3} Y_{p}^{\prime}, b_{13}=(1 / 2) \rho l^{3} Y_{r}^{\prime}-m, b_{21}=(1 / 2) \rho l^{3} K_{v}^{\prime}$, $b_{22}=(1 / 2) \rho l^{4}\left(K_{p}^{\prime}+K_{p n}^{\prime} \varepsilon(n)\right), b_{23}=(1 / 2) \rho l^{4} K_{r}^{\prime}-m z_{G}, b_{31}=$ $(1 / 2) \rho l^{3} N_{v}^{\prime}, b_{32}=(1 / 2) \rho l^{4} N_{p}^{\prime}, b_{33}=(1 / 2) \rho l^{4} N_{r}^{\prime}$, and $f=$ $-z_{G} W \phi . v, p$, and $r$ are sway velocity, roll angular rate, and yaw angular rate, respectively. $V$ is navigating speed, $M_{\text {wave }}$ is wave moment, and $M_{\text {fin }}$ is righting moment generated by zero-speed fin stabilizer. Meanings for other symbols in (A.1) can be found in [18]. Equation (A.1) is simplified into the following matrix equation:

$$
\begin{aligned}
{\left[\begin{array}{c}
\ddot{D} \\
\ddot{\phi} \\
\ddot{\psi}
\end{array}\right]=V \cdot\left[\begin{array}{lll}
a_{11} & a_{12} & a_{13} \\
a_{21} & a_{22} & a_{23} \\
a_{31} & a_{32} & a_{33}
\end{array}\right]^{-1} \cdot\left[\begin{array}{lll}
b_{11} & b_{12} & b_{13} \\
b_{21} & b_{22} & b_{23} \\
b_{31} & b_{32} & b_{33}
\end{array}\right] \cdot\left[\begin{array}{c}
\dot{D} \\
\dot{\phi} \\
\dot{\psi}
\end{array}\right] } \\
+ \\
+\left[\begin{array}{lll}
a_{11} & a_{12} & a_{13} \\
a_{21} & a_{22} & a_{23} \\
a_{31} & a_{32} & a_{33}
\end{array}\right]^{-1} \cdot\left[\begin{array}{c}
0 \\
f+M_{\text {wave }}+M_{\text {fin }} \\
0
\end{array}\right] \\
=V \cdot\left[\begin{array}{ccc}
c_{11} & c_{12} & c_{13} \\
c_{21} & c_{22} & c_{23} \\
c_{31} & c_{32} & c_{33}
\end{array}\right] \cdot\left[\begin{array}{c}
\dot{D} \\
\dot{\phi} \\
\dot{\psi}
\end{array}\right]+\left[\begin{array}{lll}
d_{11} & d_{12} & d_{13} \\
d_{21} & d_{22} & d_{23} \\
d_{31} & d_{32} & d_{33}
\end{array}\right] \\
+\left[\begin{array}{c}
f+M_{\text {wave }}+M_{\text {fin }} \\
0
\end{array}\right] .
\end{aligned}
$$


TABLE 1: Statistics of roll stabilizing performance.

\begin{tabular}{lccc}
\hline $\begin{array}{l}\text { Significant wave height } \\
(\mathrm{m})\end{array}$ & $\begin{array}{c}\text { Heading angle } \\
\left({ }^{\circ}\right)\end{array}$ & $\begin{array}{c}\text { SDRA without roll control } \\
\left({ }^{\circ}\right)\end{array}$ & $\begin{array}{c}\text { SDRA with GPC } \\
\left({ }^{\circ}\right)\end{array}$ \\
\hline$H_{s}=1$ & $\gamma=45^{\circ}$ & 2.1861 & 0.4451 \\
$H_{s}=1$ & $\gamma=90^{\circ}$ & 2.8383 & 79.64 \\
$H_{s}=1$ & $\gamma=135^{\circ}$ & 1.8226 & 0.5565 \\
\hline
\end{tabular}

TABLE 2: Calculation of energy consumption for roll stabilizing.

\begin{tabular}{|c|c|c|c|c|c|c|}
\hline $\begin{array}{l}\text { Significant wave height } \\
\text { (m) }\end{array}$ & $\begin{array}{l}\text { Heading angle } \\
\left({ }^{\circ}\right)\end{array}$ & $\begin{array}{c}\text { RSP based on } J_{0} \\
(\%)\end{array}$ & $\begin{array}{c}\text { RSP based on } J \\
(\%)\end{array}$ & $\begin{array}{c}\text { EC based on } J_{0} \\
(\mathrm{~J})\end{array}$ & $\begin{array}{c}\text { EC based on } J \\
(\mathrm{~J})\end{array}$ & $\begin{array}{l}\text { ESR } \\
(\%)\end{array}$ \\
\hline$H_{s}=1$ & $\gamma=45^{\circ}$ & 79.91 & 79.64 & 419726 & 358740 & 14.53 \\
\hline$H_{s}=1$ & $\gamma=90^{\circ}$ & 83.76 & 80.39 & 444810 & 377648 & 15.10 \\
\hline$H_{s}=1$ & $\gamma=135^{\circ}$ & 81.23 & 79.95 & 403842 & 343871 & 14.85 \\
\hline
\end{tabular}

Equation (A.2) can be further simplified into (A.3) through linearization and Laplace transformation. Consider

$$
\begin{aligned}
{\left[\begin{array}{l}
D(s) \\
\phi(s) \\
\psi(s)
\end{array}\right]=} & {\left[\begin{array}{lll}
e_{11} & e_{12} & e_{13} \\
e_{21} & e_{22} & e_{23} \\
e_{31} & e_{32} & e_{33}
\end{array}\right]^{-1} \cdot\left[\begin{array}{lll}
d_{11} & d_{12} & d_{13} \\
d_{21} & d_{22} & d_{23} \\
d_{31} & d_{32} & d_{33}
\end{array}\right] \cdot\left[\begin{array}{c}
0 \\
M_{\text {fin }} \\
0
\end{array}\right] } \\
& +\left[\begin{array}{lll}
e_{11} & e_{12} & e_{13} \\
e_{21} & e_{22} & e_{23} \\
e_{31} & e_{32} & e_{33}
\end{array}\right] \cdot\left[\begin{array}{lll}
d_{11} & d_{12} & d_{13} \\
d_{21} & d_{22} & d_{23} \\
d_{31} & d_{32} & d_{33}
\end{array}\right] \cdot\left[\begin{array}{c}
0 \\
M_{\text {wave }} \\
0
\end{array}\right] \\
= & {\left[\begin{array}{lll}
f_{11} & f_{12} & f_{13} \\
f_{21} & f_{22} & f_{23} \\
f_{31} & f_{32} & f_{33}
\end{array}\right] \cdot\left[\begin{array}{c}
0 \\
M_{\text {fin }} \\
0
\end{array}\right] } \\
& +\left[\begin{array}{lll}
f_{11} & f_{12} & f_{13} \\
f_{21} & f_{22} & f_{23} \\
f_{31} & f_{32} & f_{33}
\end{array}\right] \cdot\left[\begin{array}{c}
0 \\
M_{\text {wave }} \\
0
\end{array}\right],
\end{aligned}
$$

where $e_{11}=s^{2}-c_{11} V \cdot s, e_{12}=-c_{12} V \cdot s-d_{12} \cdot(\partial f / \partial \phi)$, $e_{13}=-c_{13} V \cdot s, e_{21}=-c_{21} V \cdot s, e_{22}=s^{2}-c_{22} V \cdot s-d_{22} \cdot(\partial f / \partial \phi)$, $e_{23}=-c_{23} V \cdot s, e_{31}=-c_{31} V \cdot s, e_{32}=-c_{32} V \cdot s-d_{32} \cdot(\partial f / \partial \phi)$, and $e_{33}=s^{2}-c_{33} V \cdot s$. From (A.3), equivalent decoupling model of ASV roll motion can be described as

$$
\phi(s)=f_{22}\left(M_{\text {fin }}+M_{\text {wave }}\right) .
$$

Corresponding ASV hydrodynamic parameters in [18] are substituted into (A.1) and expression for $f_{22}$ is obtained through derivation of (A.2) and (A.3). Consider

$$
f_{22}=\frac{0.0002416 s^{2}+0.0002044 s+0.00002912}{s^{4}+2.7629 s^{3}+2.518 s^{2}+0.8899 s+0.095} .
$$

Through data fitting of impulse response for transfer function in (A.5), higher-order roll motion model can be reduced into an equivalent second-order roll motion model given by

$$
f_{22}=\frac{0.00018319(s+0.0383)}{s^{2}+0.6426 s+0.0232} \text {. }
$$

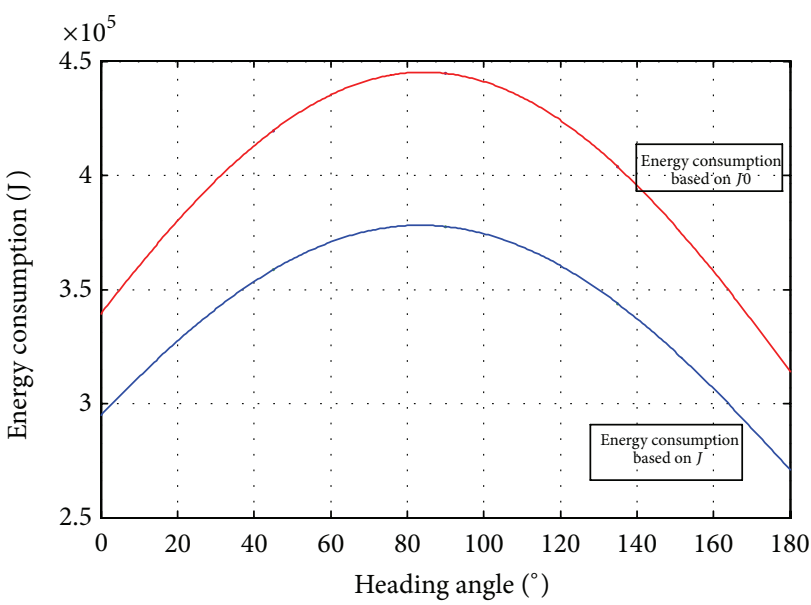

FIGURE 13: Comparison of energy consumption: $H_{s}=1$.

Considering the initial conditions, namely, $\phi^{(1)}(0)=$ $\phi(0)=0$ and $M_{\text {fin }}(0)=M_{\text {wave }}(0)=0$, (A.6) is substituted into (A.4) and then roll motion model can be expressed as a second-order differential equation through inverse Laplace transformation. Consider

$$
\begin{aligned}
\phi^{(2)}(t) & +0.6426 \phi^{(1)}(t)+0.0232 \phi(t) \\
= & 0.00018319\left[M_{\text {fin }}^{(1)}(t)+M_{\text {wave }}^{(1)}(t)\right] \\
& +0.0000070165\left[M_{\text {fin }}(t)+M_{\text {wave }}(t)\right] .
\end{aligned}
$$

Through discretization, (A.7) is transformed into a difference equation, which is predictive model of GPC and obtained by applying Euler approximation method. Equivalent difference equation for roll motion is given by

$$
\begin{aligned}
\phi(k) & -1.5947 \phi(k-1)+0.6088 \phi(k-2) \\
= & 1.1152 \times 10^{-4}\left[M_{\text {fin }}(k)+M_{\text {wave }}(k)\right] \\
& -1.0725 \times 10^{-4}\left[M_{\text {fin }}(k-1)+M_{\text {wave }}(k-1)\right] .
\end{aligned}
$$




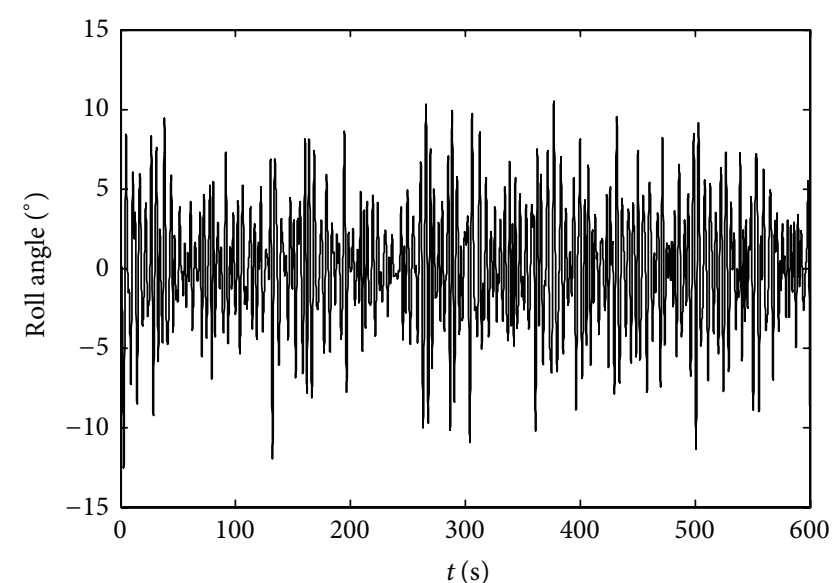

FIGURE 14: Simulation without roll reduction when $H_{s}=1.2$ and $\gamma=90^{\circ}$.

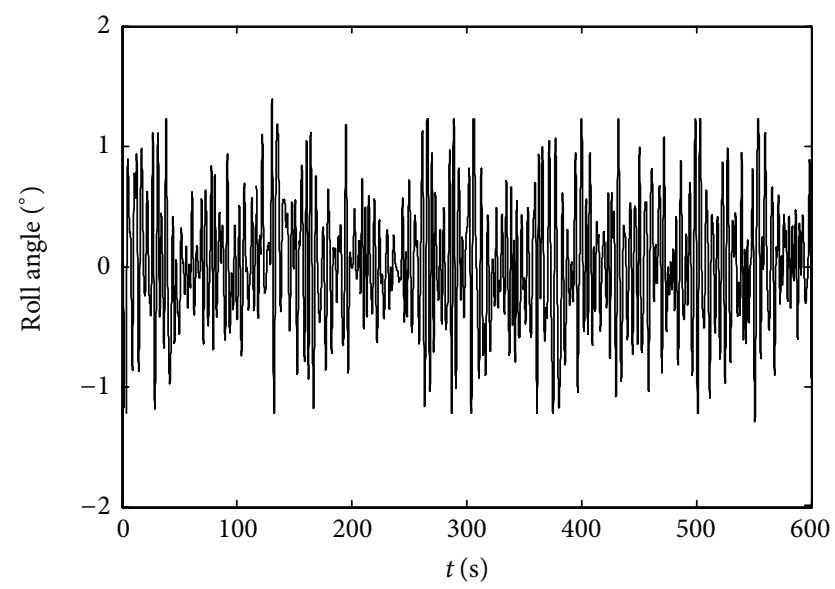

FIGURE 15: Simulation of self-adaptive GPC when $H_{s}=1.2$ and $\gamma=$ $90^{\circ}$.

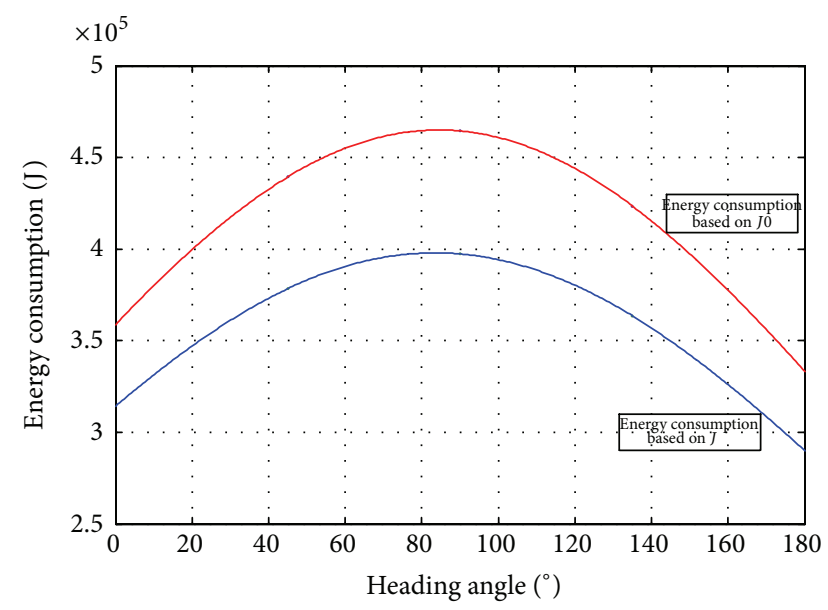

FIGURE 16: Comparison of energy consumption: $H_{s}=1.2$.

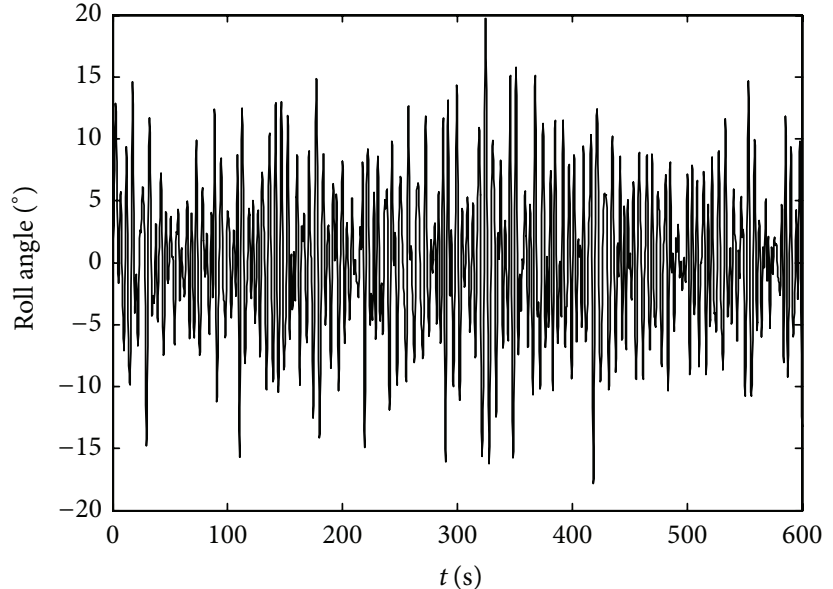

FIGURE 17: Simulation without roll reduction when $H_{s}=1.5$ and $\gamma=90^{\circ}$.

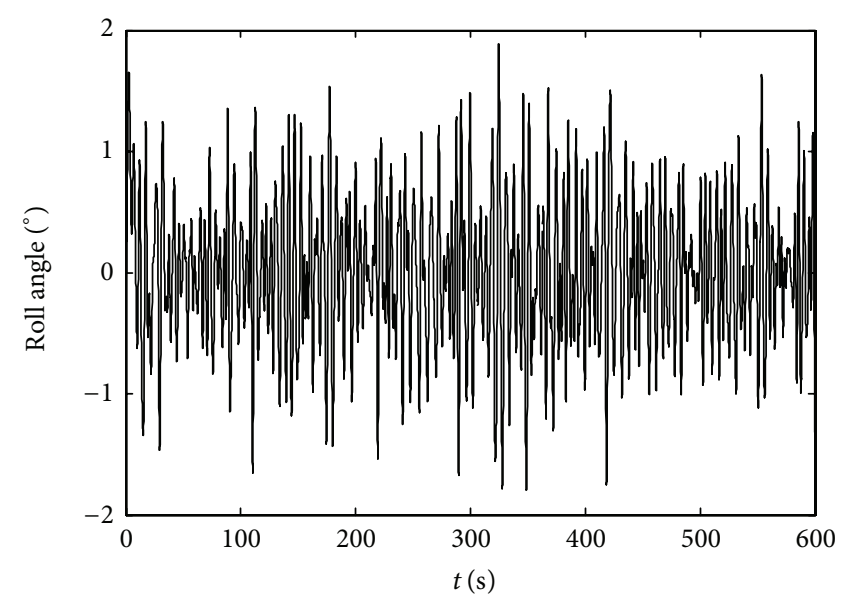

FIGURE 18: Simulation of self-adaptive GPC when $H_{s}=1.5$ and $\gamma=$ $90^{\circ}$.

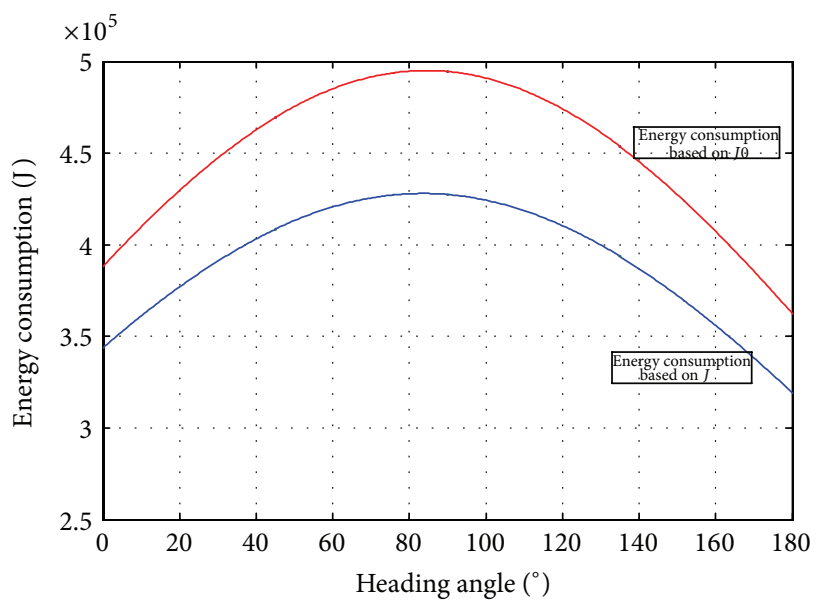

FIGURE 19: Comparison of energy consumption: $H_{s}=1.5$. 


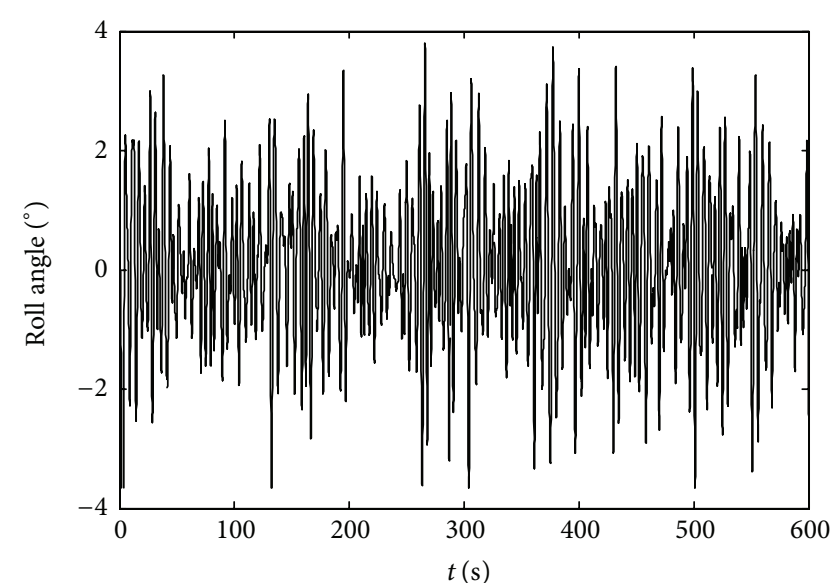

FIGURE 20: Simulation of traditional design when $H_{s}=1.2$ and $\gamma=$ $90^{\circ}$.

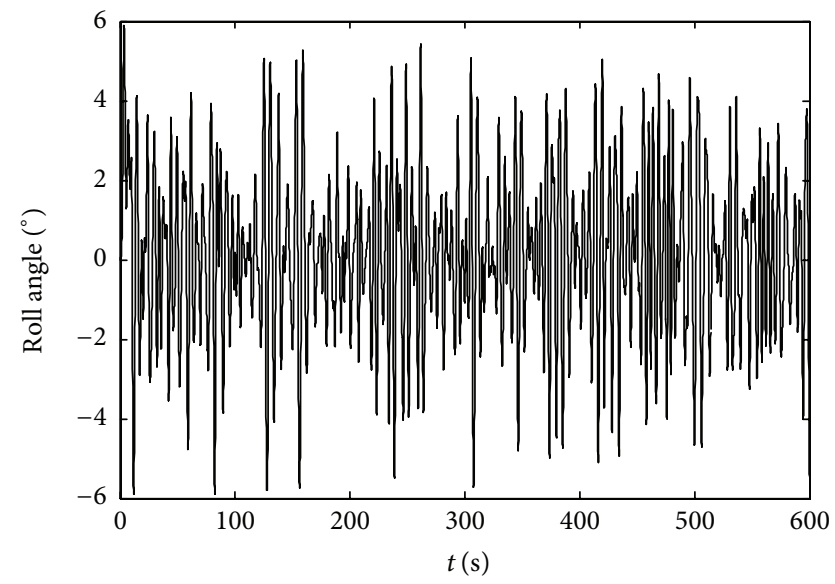

FIgURE 21: Simulation of traditional design when $H_{s}=1.5$ and $\gamma=$ $90^{\circ}$.

Here, sampling period for discretization is $1 \mathrm{~s}$. In the above deduction, coupling effect of yaw and sway on roll is considered adequately. So, (A.8) is an equivalent equation for roll motion.

\section{B. Coefficient Calculation of Diophantine Equations}

If $\bar{A}\left(z^{-1}\right)=A\left(z^{-1}\right) \Delta=1+\bar{a}_{1} z^{-1}+\bar{a}_{2} z^{-2}+\bar{a}_{3} z^{-3}$, the following formulas for recursive calculations are obtained:

$$
\begin{gathered}
e_{j}=f_{0}^{j}=F_{j}(0) \\
f_{i}^{j+1}=f_{i+1}^{j}-\bar{a}_{i+1} f_{0}^{j} \quad(0 \leq i<2) \\
f_{2}^{j+1}=-\bar{a}_{3} f_{0}^{j} \\
g_{j}=e_{j} b_{0}+h_{0}^{j} \\
h_{0}^{j+1}=e_{j} b_{1} .
\end{gathered}
$$

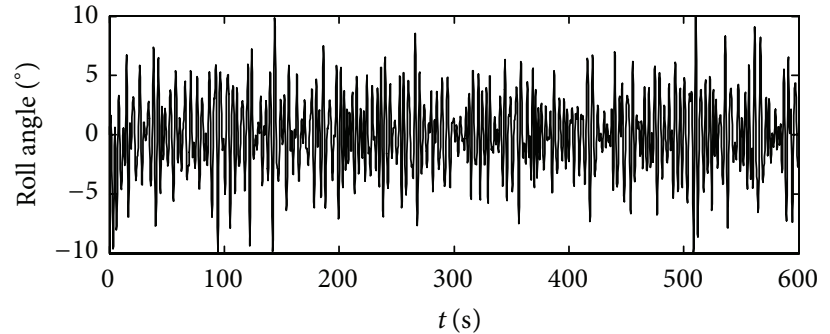

FIGURE 22: Simulation without roll reduction under uncertainty conditions.

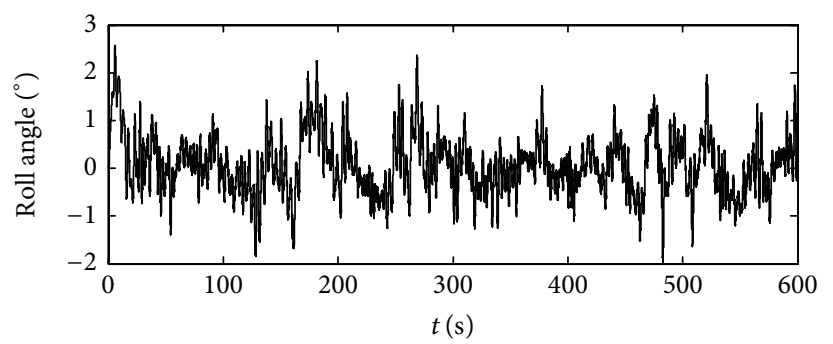

FIGURE 23: Simulation of self-adaptive GPC under uncertainty conditions.

According to Diophantine equations (9)-(10), initial values for recursive calculations are

$$
\begin{gathered}
E_{1}\left(z^{-1}\right)=e_{0}=1 \\
F_{1}\left(z^{-1}\right)=z\left[1-\bar{A}\left(z^{-1}\right)\right]=-\bar{a}_{1}-\bar{a}_{2} z^{-1}-\bar{a}_{3} z^{-2} \\
G_{1}\left(z^{-1}\right)=g_{0}=e_{0} b_{0} \\
H_{1}\left(z^{-1}\right)=z\left(e_{0} B\left(z^{-1}\right)-e_{0} b_{0}\right)=b_{1} .
\end{gathered}
$$

\section{Parameter Estimation of Roll Motion Model}

Consider the case of time-variant parameters in ASV roll motion model; namely,

$$
\bar{A}\left(z^{-1}, k\right) \phi(k)=\bar{B}\left(z^{-1}, k\right) M_{\text {fin }}(k)+\bar{B}\left(z^{-1}, k\right) M_{\text {wave }}(k)
$$

where

$$
\begin{gathered}
\bar{A}\left(z^{-1}, k\right)=A\left(z^{-1}, k\right) \Delta \\
=1+\bar{a}_{1}(k) z^{-1}+\bar{a}_{2}(k) z^{-2}+\bar{a}_{3}(k) z^{-3} \\
\bar{B}\left(z^{-1}, k\right)=B\left(z^{-1}, k\right) \Delta=\bar{b}_{0}(k)+\bar{b}_{1}(k) z^{-1}+\bar{b}_{2}(k) z^{-2} .
\end{gathered}
$$




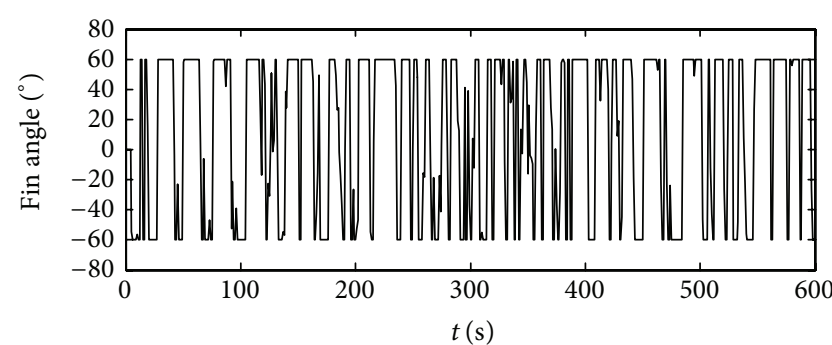

FIGURE 24: Fin angle of self-adaptive GPC under uncertainty conditions.

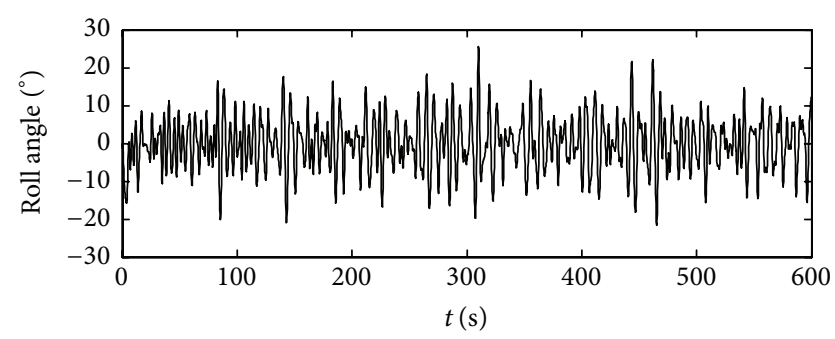

FIGURE 25: Simulation without roll reduction when $H_{s}=3$ and $\gamma=$ $90^{\circ}$.

If model parameters and data parameters are expressed as the following vectors respectively, namely,

$$
\begin{gathered}
\theta(k)=\left[\bar{a}_{1}(k), \bar{a}_{2}(k), \bar{a}_{3}(k), \bar{b}_{0}(k), \bar{b}_{1}(k), \bar{b}_{2}(k)\right]^{T}, \\
X(k)=\left[-\phi(k-1),-\phi(k-2),-\phi(k-3), M_{\text {fin }}(k),\right. \\
\left.M_{\text {fin }}(k-1), M_{\text {fin }}(k-2)\right]^{T},
\end{gathered}
$$

(C.1) can be transformed into

$$
\phi(k)=\theta^{T}(k) \cdot X(k)+\bar{B}\left(z^{-1}, k\right) M_{\text {wave }}(k) .
$$

Parameter vector $\theta(k)$ is estimated with recursive least square method and coefficients of corresponding terms in $A\left(z^{-1}\right)$ and $B\left(z^{-1}\right)$ are obtained. Then, recursive calculations are performed according to (B.1). $E_{j}\left(z^{-1}\right), F_{j}\left(z^{-1}\right), G_{j}\left(z^{-1}\right)$, and $H_{j}\left(z^{-1}\right)$ are substituted into (25); as a result, optimal control variable $M_{\text {fin }}(k+1)$ can be calculated.

Algorithm for estimating parameter vector with recursive least square method is given by

$\theta(k+1)$

$$
=\theta(k)+\frac{\Omega(k-1) \cdot X(k) \cdot\left[\phi(k)-\theta^{T}(k) \cdot X(k)\right]}{\mu+X^{T}(k) \cdot \Omega(k-1) \cdot X(k)}
$$

$\Omega(k)$

$$
=\frac{1}{\mu} \cdot\left[\Omega(k-1)-\frac{\Omega(k-1) \cdot X(k) \cdot X^{T}(k) \cdot \Omega(k-1)}{\mu+X^{T}(k) \cdot \Omega(k-1) \cdot X(k)}\right],
$$

where $\mu$ is forgetting factor, $0<\mu<1$, and $\Omega(k)$ is a positive definite matrix.

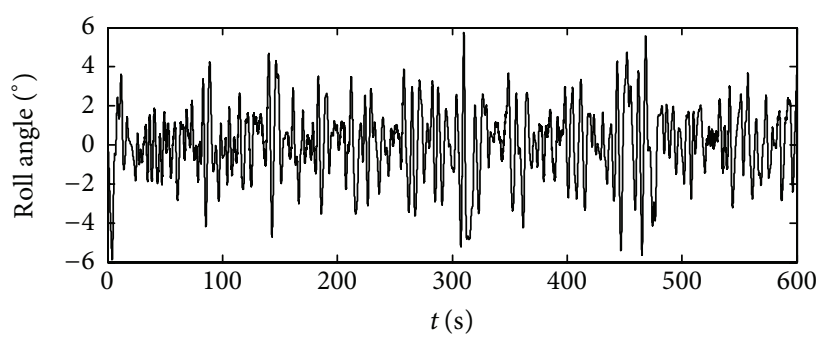

FIGURE 26: Simulation of self-adaptive GPC when $H_{s}=3$ and $\gamma=$ $90^{\circ}$.

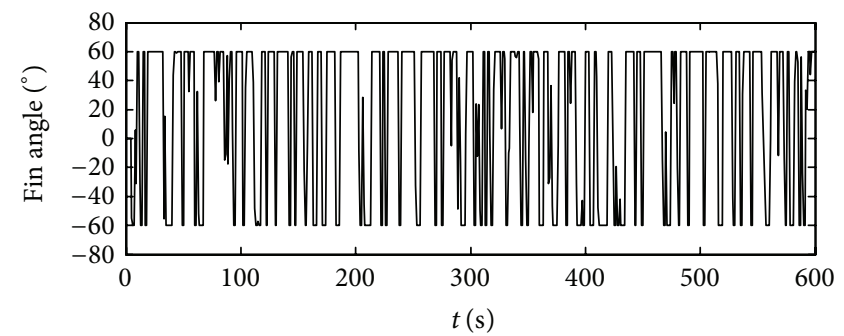

FIGURE 27: Fin angle of self-adaptive GPC when $H_{s}=3$ and $\gamma=90^{\circ}$.

\section{Model in Traditional Design of Roll Stabilizing System}

Considering coupling effects of sway and yaw on roll, which are often disregarded in traditional design of ASV roll stabilizing system, a simplified roll motion model is obtained on the basis of ASV coupling horizontal model and is given by

$$
a_{22} \dot{p}=V b_{22} p+f+M_{\text {wave }}+M_{\text {fin }}
$$

namely,

$$
\begin{aligned}
& a_{22} \phi^{(2)}(t)-V b_{22} \phi^{(1)}(t)+z_{G} W \phi(t) \\
& =M_{\text {wave }}(t)+M_{\text {fin }}(t) .
\end{aligned}
$$

Relevant parameters are substituted into (D.2), and then (D.3) is obtained through further derivation. Consider

$$
\begin{aligned}
& \phi^{(2)}(t)+1.8378 \phi^{(1)}(t)+0.7889 \phi(t) \\
& \quad=2.4219 \times 10^{-4}\left[M_{\text {wave }}(t)+M_{\text {fin }}(t)\right] .
\end{aligned}
$$

Through discretization, (D.3) is transformed into a difference equation, which can be regarded as the predictive model of GPC, and is obtained by applying Euler approximation method. Equivalent difference equation for roll motion is given by

$$
\begin{gathered}
\phi(k)-1.0582 \phi(k-1)+0.2757 \phi(k-2) \\
=6.678 \times 10^{-5}\left[M_{\text {wave }}(k)+M_{\text {fin }}(k)\right] .
\end{gathered}
$$

Here, sampling period for discretization is $1 \mathrm{~s}$. In the above deduction, coupling effect of yaw and sway on roll is disregarded. So, (D.4) can be used as the roll motion model in traditional design of ASV roll stabilizing system. 


\section{Abbreviations}

ASV: Autonomous surface vehicle

GPC: General predictive control

RSP: Roll stabilizing performance

SDRA: Standard deviation of roll angle

ESR: Energy-saving ratio

EC: Energy consumption.

\section{Symbols}

\%: $\quad$ Yaw angle

D: $\quad$ Sway displacement

$\phi$ : $\quad$ Roll angle

$\widehat{\psi}: \quad$ Measured value of $\psi$

$\widehat{D}: \quad$ Measured value of $D$

$\widehat{\phi}: \quad$ Measured value of $\phi$

$e_{0}: \quad$ Span length

$\rho: \quad$ Sea water density

$C_{d}$ : Coefficient of drag force

$k$ : $\quad$ Proportion factor

2a: Chord length

$c$ : $\quad$ Distance from fin axis to midpoint of chord length

$\omega^{\prime}$ : $\quad$ Angular rate of fin

$J_{I}: \quad$ Additional moment of inertia

$d$ : $\quad$ Distance from fin axis to the midpoint where force on additional mass acts

$c_{1}$ : Constant

$c_{2}: \quad$ Constant

$L_{\text {lift }}: \quad$ Lift force

$L_{\text {zero }}$ : Lift force under zero-speed condition

$\Delta L_{\text {lift }}:$ Additional lift force

$V: \quad$ Navigating speed

$w$ : $\quad$ Future reference value

$J: \quad$ Performance index for optimization

$G(z): \quad$ General control process

u: $\quad$ Input of $G(z)$

$y: \quad$ Output of $G(z)$

$\widetilde{G}(z): \quad$ Model of $G(z)$

$e: \quad$ Future error of GPC

E: $\quad$ Mathematical expectation

$N_{1}$ : $\quad$ Minimal time domain of GPC prediction

$\mathrm{N}_{2}$ : $\quad$ Maximal time domain of GPC prediction

$\phi_{r}: \quad$ Expected value of $\phi$

$\lambda$ : Weight coefficient of performance index

$N_{u}: \quad$ Control time domain

$M_{\text {fin }}$ : Righting moment generated by fin stabilizer

$\alpha: \quad$ Fin angle

$T$ : $\quad$ Sampling period

$A\left(z^{-1}\right)$ : Coefficient polynomial of roll motion model

$B\left(z^{-1}\right)$ : Coefficient polynomial of roll motion model

$E_{j}$ : Coefficient polynomial of Diophantine equation

\begin{tabular}{|c|c|}
\hline$F_{j}:$ & $\begin{array}{l}\text { Coefficient polynomial of Diophantine } \\
\text { equation }\end{array}$ \\
\hline$G_{j}:$ & $\begin{array}{l}\text { Coefficient polynomial of Diophantine } \\
\text { equation }\end{array}$ \\
\hline$H_{j}:$ & $\begin{array}{l}\text { Coefficient polynomial of Diophantine } \\
\text { equation }\end{array}$ \\
\hline $\operatorname{abs}(\cdot)$ : & $\begin{array}{l}\text { Matrix composed of absolute values of } \\
\text { original matrix elements }\end{array}$ \\
\hline $\operatorname{sign}(\cdot):$ & Signum function \\
\hline$l_{f}:$ & Lever of lift force \\
\hline$\dot{J}_{0}:$ & $\begin{array}{l}\text { Performance index of GPC considering } \\
\text { RSP instead of EC }\end{array}$ \\
\hline$H_{s}:$ & Significant wave height \\
\hline$\gamma:$ & Heading angle \\
\hline$\mu:$ & Forgetting factor \\
\hline$\Omega:$ & Positive definite matrix \\
\hline$\theta:$ & Vector of model parameters \\
\hline$X:$ & Vector of data parameters \\
\hline$v:$ & Sway velocity \\
\hline$p:$ & Roll angular rate \\
\hline$r:$ & Yaw angular rate \\
\hline$M_{\text {wave }}$ : & Wave moment \\
\hline$m:$ & AUV mass \\
\hline$l:$ & Total length of AUV \\
\hline$x_{G}, y_{G}, z_{G}:$ & $\begin{array}{l}\text { Coordinates of gravity center position } \\
\text { in vehicle coordinate system }\end{array}$ \\
\hline$I_{x}, I_{y}, I_{z}:$ & $\begin{array}{l}\text { Moments of inertia around the } x \text {-axis, } \\
y \text {-axis, and } z \text {-axis }\end{array}$ \\
\hline$I_{x y}, I_{y z}, I_{z x}$ & $\begin{array}{l}\text { Products of inertia for XOY plane, YOZ } \\
\text { plane, and XOZ plane }\end{array}$ \\
\hline$Y_{(\cdot)}^{\prime}, K_{(\cdot)}^{\prime}, N_{(\cdot)}^{\prime}:$ & Coefficients of hydrodynamic force \\
\hline$W:$ & AUV self-weight \\
\hline$\varepsilon(n)$ & $\begin{array}{l}\text { Parameter related to propeller } \\
\text { rotational rate. }\end{array}$ \\
\hline
\end{tabular}

\section{Conflict of Interests}

The authors declare that there is no conflict of interests regarding the publication of this paper.

\section{Acknowledgments}

The project is financially supported by the National Natural Science Foundation (NNSF) of China under Grant E091002/50979017, Ph.D. Programs Foundation of Ministry of Education of China and Basic Technology under Grant 20092304110008, Research Operation Item Foundation of Central University under Grant HEUCFZ 1026, and Harbin Science and Technology Innovation Talents of Special Fund Project (Outstanding Subject Leaders) under Grant 2012RFXXG083.

\section{References}

[1] M.-C. Fang, P.-E. Chang, and J.-H. Luo, "Wave effects on ascending and descending motions of the autonomous underwater vehicle," Ocean Engineering, vol. 33, no. 14-15, pp. 19721999, 2006. 
[2] G. N. Roberts, "Trends in marine control systems," Annual Reviews in Control, vol. 32, no. 2, pp. 263-269, 2008.

[3] R. P. Dallinga, "Roll stabilization of motor yacht: hydrodynamic aspects of the comparison of anti-roll tanks and fins," 2002,http://www.marin.nl/web/News/News-items/Rollstabilization-of-motor-yacht-Hydrodynamic-aspects-of-thecomparison-of-antiroll-tanks-and-fins.htm.

[4] W. Fan, J. Hongzhang, and Q. Zhigang, "Modeling for active fin stabilizers at zero speed," Ocean Engineering, vol. 36, no. 17-18, pp. 1425-1437, 2009.

[5] H.-Z. Jin, L.-X. Pan, and A.-L. Zou, "Rolling model of surface vehicle and its stabilizing control," Journal of Dalian Maritime University, vol. 36, no. 1, pp. 1-4, 2010 (Chinese).

[6] M. J. Logsdon, Coupled roll and directional stability characteristics of surface ships [Ph.D. dissertation], Naval Postgraduate School, 1992.

[7] F. Carravetta, G. Felici, and P. Palumbo, "Regulation of a manned sea-surface vehicle via stochastic optimal control," in Proceedings of the 17th World Congress, International Federation of Automatic Control (IFAC '08), Seoul, South Korea, July 2008.

[8] E. Sahler, Analysis of a single-degree-of-freedom roll motion model: simulation, sensitivity study, and comparison to multidegree-of-freedom models [Ph.D. dissertation], Oregon State University, 1996.

[9] H. Z. Jin, L. X. Pan, and L. L. Wang, "Adaptive variable structure control with neuron for low speed stabilizing of submarine near surface," Control and Decision, vol. 25, no. 4, pp. 562-571, 2010 (Chinese).

[10] H. Z. Jin, L. X. Pan, and L. L. Wang, "Modified variable structure control in rudder roll damping of submarine near free-surface," Journal of Harbin Institute of Technology, vol. 42, no. 9, pp. 14621466, 2010 (Chinese).

[11] X. Peng, X. Yang, and X. Zhao, "On-line adaptive neural networks for ship motion control," in Proceedings of the IEEE/RSJ International Conference on Intelligent Robots and Systems (IROS '07), pp. 3592-3597, San Diego, Calif, USA, OctoberNovember 2007.

[12] J. V. Salcedo and M. Martínez, "GPC robust design using linear and/or bilinear matrix inequalities," European Journal of Control, vol. 13, no. 5, pp. 451-472, 2007.

[13] M.-C. Fang and J.-H. Luo, "On the track keeping and roll reduction of the ship in random waves using different sliding mode controllers," Ocean Engineering, vol. 34, no. 3-4, pp. 479488, 2007.

[14] T. I. Fossen, Guidance and Control of Ocean Vehicles, John Wiley and Sons, NewYork, NY, USA, 1994.

[15] M. H. Sohn and J. W. Chang, "Flow visualization and aerodynamic load calculation of three types of clap-fling motions in a Weis-Fogh mechanism," Aerospace Science and Technology, vol. 11, no. 2-3, pp. 119-129, 2007.

[16] H.-Z. Jin, Y.-M. Luo, Z.-G. Qi, and Z.-C. Yang, "Investigation of the lifting force of a zero speed fin stabilizer with Weis-Fogh mechanism," Journal of Harbin Engineering University, vol. 28, no. 7, pp. 762-767, 2007 (Chinese).

[17] X. F. Zhang and H. Z. Jin, Fuzzy Control of Fin Stabilizer at Zero Speed Based on Improved Genetic Algorithm, ISKE, Chengdu, China, 2007.

[18] Y. M. Luo, Research on fin stabilizers at zero speed and its electro-servo system for ship [Ph.D. thesis], Harbin Engineering University, 2007 (Chinese).
[19] J. Ringwood, "The dynamics of wave energy," in Proceedings of the Irish Signals and Systems Conference, Dublin, Ireland, June 2006.

[20] L. X. Pan, H. Z. Jin, and L. L. Wang, "Direct self-adaptive general predictive control for roll stabilizing of autonomous surface vehicle," in Proceedings of the 3rd International Conference on Modelling and Simulation, Wuxi, China, June 2010.

[21] X. F. Zhang, Research on modeling and control of fin stabilizer at zero speed [Ph.D. dissertation], Harbin Engineering University, 2008. 


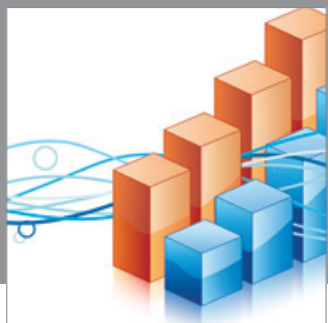

Advances in

Operations Research

mansans

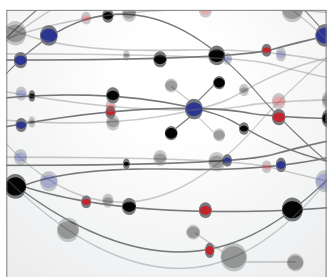

The Scientific World Journal
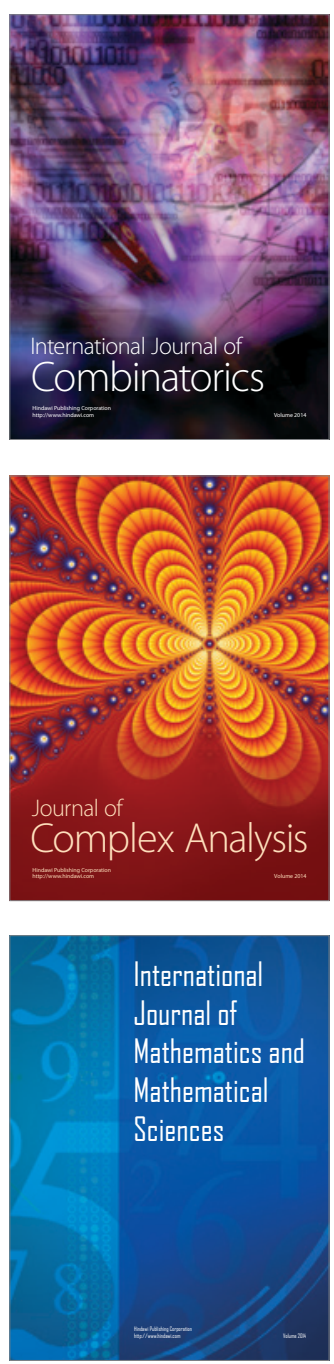
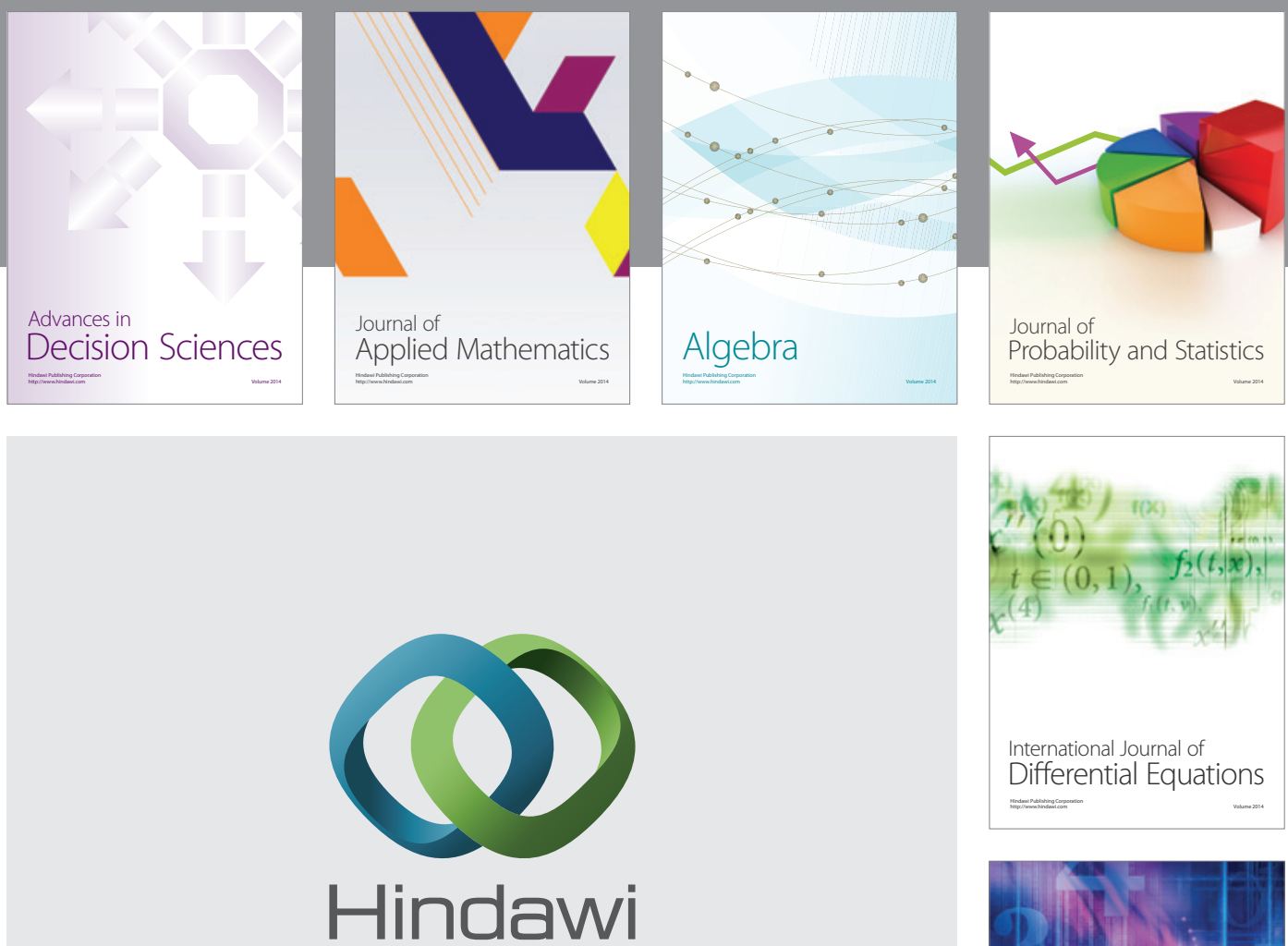

Submit your manuscripts at http://www.hindawi.com
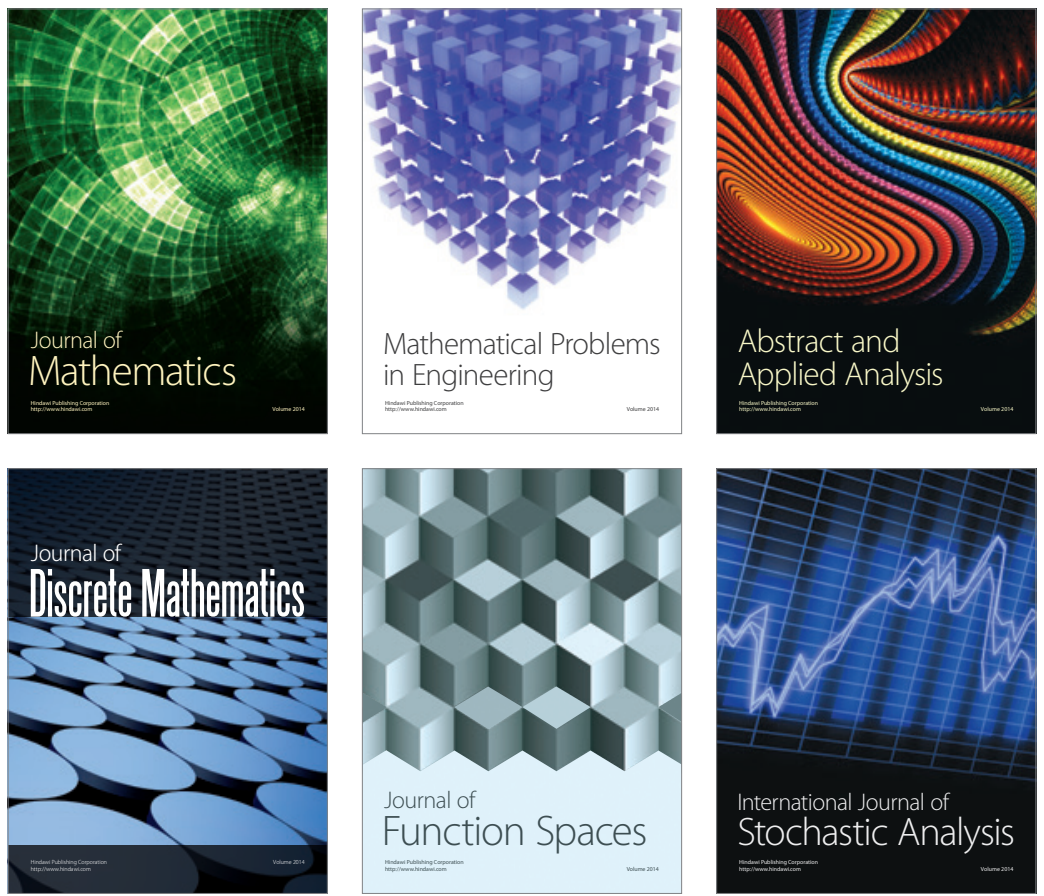

Journal of

Function Spaces

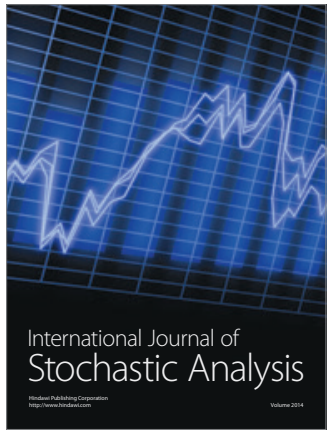

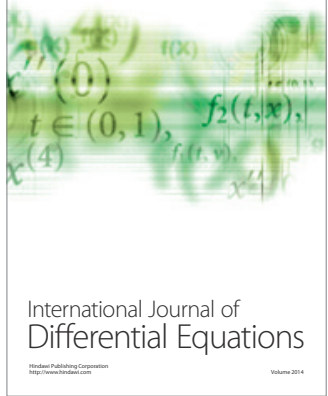
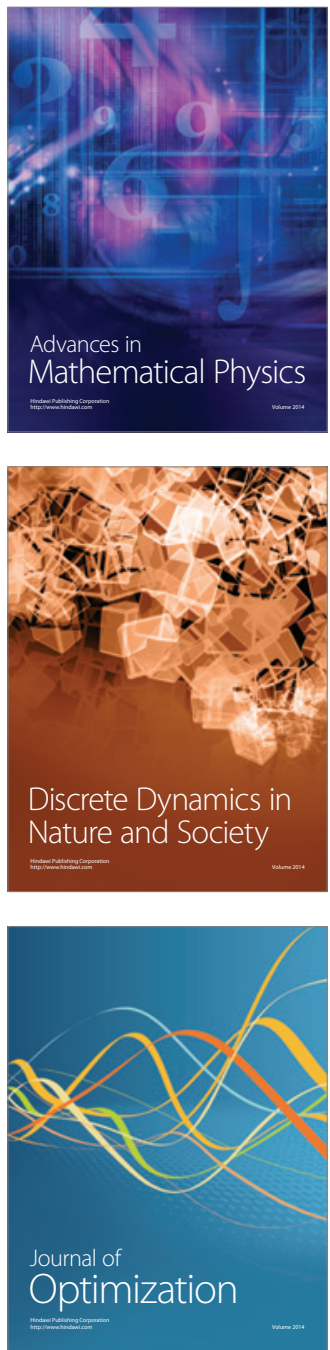